\title{
Solutions for a Class of Nonperiodic Superquadratic Hamiltonian Elliptic Systems Involving Gradient Terms
}

\author{
Shengzhong Duan ${ }^{1}$ and Xian $\mathrm{Wu}^{2}$ \\ ${ }^{1}$ Department of Mathematics, Baoshan University, Baoshan, Yunnan 678000, China \\ ${ }^{2}$ Department of Mathematics, Yunnan Normal University, Kunming, Yunnan 650092, China \\ Correspondence should be addressed to Shengzhong Duan; duanshengzhong@163.com
}

Received 22 January 2017; Accepted 8 March 2017; Published 28 March 2017

Academic Editor: Gabriele Bonanno

Copyright (c) 2017 Shengzhong Duan and Xian Wu. This is an open access article distributed under the Creative Commons Attribution License, which permits unrestricted use, distribution, and reproduction in any medium, provided the original work is properly cited.

In the present paper, we consider the following Hamiltonian elliptic system (HES): $-\Delta u+b(x) \cdot \nabla u+V(x) u=H_{v}(x, u, v), x \in \mathbb{R}^{N}$, $-\Delta v-b(x) \cdot \nabla v+V(x) v=H_{u}(x, u, v), x \in \mathbb{R}^{N}$. A new existence result of nontrivial solutions is obtained for the system (HES) via variational methods for strongly indefinite problems, which generalizes some known results in the literatures.

\section{Introduction and Main Results}

The goal of this paper is to study the existence of solutions for the nonperiodic elliptic systems in Hamiltonian form

$$
\begin{aligned}
-\Delta u+b(x) \cdot \nabla u+V(x) u=H_{v}(x, u, v), & \\
& x \in \mathbb{R}^{N}, \\
-\Delta v-b(x) \cdot \nabla v+V(x) v=H_{u}(x, u, v), & \\
& x \in \mathbb{R}^{N},
\end{aligned}
$$

where $z=(u, v): \mathbb{R}^{N} \rightarrow \mathbb{R}^{1} \times \mathbb{R}^{1}, b=\left(b_{1}, \ldots, b_{N}\right) \in$ $C^{1}\left(\mathbb{R}^{N}, \mathbb{R}^{N}\right), V(x) \in C\left(\mathbb{R}^{N}, \mathbb{R}\right)$, and $H \in C^{1}\left(\mathbb{R}^{N} \times \mathbb{R}^{2}, \mathbb{R}\right)$. Such a system arises when one is looking for stationary solutions to certain systems of optimal control (Lions [1]) or systems of diffusion equations (Itô [2] and Nagasawa [3]).

In recent years, the systems like or similar to (HES) in the whole space $\mathbb{R}^{N}$ were studied by a number of authors. Most of these works focused on the case $b(x)=0$. An usual way to overcome this difficulty is to consider the corresponding functional in the space of radially symmetric functions. In this way, De Figueiredo and Yang [4] considered the system

$$
\begin{array}{cc}
-\Delta u+u=H_{v}(x, u, v) & \text { in } \mathbb{R}^{N}, \\
-\Delta v+v=H_{u}(x, u, v) & \text { in } \mathbb{R}^{N},
\end{array}
$$

where $H(x, u, v)=F(x, u)+G(x, v), F(x, u)=\int_{0}^{u} f(x, t) d t$, and $G(x, v)=\int_{0}^{v} g(x, t) d t$. They proved that system (1) has a radial solution pair under the assumptions that $f(x, t)$ and $g(x, t)$ are superlinear in $t$ and radially symmetric with respect to $x,|f(x, t)| \leq c\left(1+|t|^{p-1}\right)$ and $|g(x, t)| \leq c\left(1+|t|^{q-1}\right)$ with $2 \leq p, q<2 N /(N-2), N>2$. This result was later generalized by Sirakov [5] to the system

$$
\begin{aligned}
& -\Delta u+b(x) u=H_{v}(x, u, v) \quad \text { in } \mathbb{R}^{N}, \\
& -\Delta v+b(x) v=H_{u}(x, u, v) \quad \text { in } \mathbb{R}^{N} .
\end{aligned}
$$

In [6], Zhao et al. considered periodic asymptotically linear elliptic systems

$$
\begin{aligned}
-\Delta u+V(x) u & =H_{v}(x, u, v) \quad \text { in } \mathbb{R}^{N}, \\
-\Delta v+V(x) v & =H_{u}(x, u, v) \quad \text { in } \mathbb{R}^{N}, \\
u(x) & \longrightarrow 0, \\
v(x) & \longrightarrow 0
\end{aligned}
$$

$$
\text { as }|x| \longrightarrow \infty \text {, }
$$

where the potential $V$ is periodic and has a positive bound from below and $f(x, t)$ and $g(x, t)$ are periodic in $x$, asymptotically linear in $t$ as $|t| \rightarrow \infty$. By using critical point theory 
of strongly indefinite functionals, they obtained the existence of a positive ground state solution as well as infinitely many geometrically distinct solution for systems (3) under the assumptions that $f(x, t)$ and $g(x, t)$ are odd in $t$. For other results, we refer readers to [7-17].

Without assumption of periodicity or radially symmetric about nonlinearities, the problem is quite different in nature and there has not been much work done up to now. In a recent paper [18], Wang et al. considered the following nonperiodic elliptic systems in Hamiltonian form:

$$
\begin{aligned}
-\Delta u+V(x) u & =H_{v}(x, u, v) \text { in } \mathbb{R}^{N}, \\
-\Delta v+V(x) v & =H_{u}(x, u, v) \text { in } \mathbb{R}^{N}, \\
u(x) & \longrightarrow 0, \\
v(x) & \longrightarrow 0
\end{aligned}
$$

as $|x| \longrightarrow \infty$

and obtained the following theorem.

Theorem A (see [18, Theorem 1.1]). Suppose that the following conditions are satisfied:

$\left(V_{0}\right) V(x)=1$.

$\left(H_{0}\right)$ There is $\mu>2$ such that $0<\mu H(x, z) \leq H_{z}(x, z) z$ if $z \neq 0$.

$\left(H_{1}\right) H(x, z) \in C^{1}\left(\mathbb{R}^{N} \times \mathbb{R}^{2}, \mathbb{R}\right), H_{z}(x, z)=h(x,|z|) z$, where $h: \mathbb{R}^{N} \times \mathbb{R}^{+} \rightarrow \mathbb{R}$ with $h(x, s) \geq 0, h(x, s)=$ $o(1)$ as $s \rightarrow 0$ uniformly in $x$, and there exist $p \in$ $\left[\mu, 2^{*}\right), c_{1}>0$ such that $h(x, s) \leq c_{1}\left(1+s^{p-2}\right)$, where $2^{*}=2 N /(N-2)$.

$\left(H_{2}\right)$ There is $h_{\infty} \in C^{1}\left(\mathbb{R}^{+}, \mathbb{R}^{+}\right)$with $h_{\infty}^{\prime}(s)>0$ for $s>$ 0 , and $h(x, s) \rightarrow h_{\infty}(s)$ as $|x| \rightarrow+\infty$ uniformly in bounded set of $s, h_{\infty}(s) \leq h(x, s)$ and $H(x, z)-$ $H_{\infty}(z) \geq b(x)|z|^{\mu}$ for all $x \in \mathbb{R}^{N}$, where $b(x) \neq$ $0, b(x) \geq 0$ and $H_{\infty}(z)=\int_{0}^{|z|} h_{\infty}(s) s d s$.

Then system (4) has one solution.

In the present paper, we are interested in the existence of solutions for Hamiltonian-elliptic systems (HES) involving gradient terms and nonperiodic superquadratic nonlinearities. The class of problems treated here has several difficulties. First, the problem is set on $\mathbb{R}^{N}$; a main difficulty when dealing with this problem is the lack of compactness of the Sobolev embedding theorem. Second, the variational functional is strongly indefinite. Therefore, the classic critical point theorem cannot be applied directly. Third, the nonlinearities $H(x, u, v)$ are nonperiodic in variable $x$ and superquadratic at infinity; the method in [6] cannot be applied to obtain the existence of solutions. Finally, the appearance of the gradient terms in the systems also brings us some difficulties; in this case, the variational framework in [18] cannot work any longer. Inspired by recent works of Zhao and Ding [19], we are going to investigate the existence of solutions for the Hamiltonian elliptic systems (HES). By using the critical point theory of strongly indefinite functional which was developed recently by Bartsch and Ding [10, 20] and the reduction methods which was developed in [21, 22], we obtain an existence result of problem (HES), which generalizes Theorem A.

Our fundamental assumptions are as follows:

$\left(V_{1}\right) V \in C\left(\mathbb{R}^{N}, \mathbb{R}\right)$ is 1-periodic in $x_{i}$ for $i=1, \ldots, N$ and $V_{0}:=\min _{x \in \mathbb{R}^{N}} V(x)>0$.

$\left(B_{0}\right) b \in C^{1}\left(\mathbb{R}^{N}, \mathbb{R}^{N}\right)$, and $\operatorname{div}(b)=0$.

$\left(B_{1}\right) b$ is 1-periodic in $x_{i}$ for $i=1, \ldots, N$.

$\left(H_{3}\right) \quad H(x, z) \in C^{1}\left(\mathbb{R}^{N} \times \mathbb{R}^{2},[0,+\infty)\right), H_{z}(x, z)=h(x$, $|z|) z$, where $h: \mathbb{R}^{N} \times \mathbb{R}^{+} \rightarrow \mathbb{R}$ with $h(x, s) \geq$ $0, h(x, s)=o(1)$ as $s \rightarrow 0$ uniformly in $x$, and there exist $p \in\left(2,2^{*}\right), c_{1}>0$ such that $h(x, s) \leq c_{1}\left(1+s^{p-2}\right)$, where $2^{*}=2 N /(N-2)$.

$\left(H_{4}\right) H(x, z) /|z|^{2} \rightarrow+\infty$ as $|z| \rightarrow+\infty$ uniformly in $x$.

$\left(H_{5}\right) \inf _{x \in \mathbb{R}^{N}} \widetilde{H}(x, z)>0$ whenever $z \neq 0$, where $\widetilde{H}(x, z)=$ $(1 / 2) H_{z}(x, z) z-H(x, z)$.

$\left(H_{6}\right)\left|H_{z}(x, z)\right|^{\tau} \leq c_{2} \widetilde{H}(x, z)|z|^{\tau}$ for some $c_{2}>0, \tau>$ $\max \{1, N / 2\}$ and all $(x, z)$ with $|z|$ large enough.

$\left(H_{7}\right)$ There is $h_{\infty} \in C^{1}\left(\mathbb{R}^{+}, \mathbb{R}^{+}\right)$with $h_{\infty}^{\prime}(s)>0$ for $s>$ 0 , and $h(x, s) \rightarrow h_{\infty}(s)$ as $|x| \rightarrow+\infty$ uniformly in bounded set of $s, h_{\infty}(s) \leq h(x, s)$ for all $(x, s) \in \mathbb{R}^{N} \times$ $\mathbb{R}^{+}$and $h_{\infty}(s)<h(x, s)$ whenever $s>0$.

Now we can state our main result.

Theorem 1. Let $\left(V_{1}\right),\left(B_{0}\right),\left(B_{1}\right)$, and $\left(H_{3}\right)-\left(H_{7}\right)$ be satisfied. Then system (HES) has at least one nontrivial solution.

Remark 2. Theorem 1 extends and improves Theorem A. First, we only need to assume that the potential $V(x)$ is periodic and has a positive bound from below. Second, the conditions $\left(\mathrm{H}_{4}\right)$ and $\left(\mathrm{H}_{5}\right)$ can be obtained by $\left(\mathrm{H}_{0}\right)$ and $\left(\mathrm{H}_{3}\right)$. In fact, by $\left(\mathrm{H}_{3}\right)$, we know that

$$
\left|H_{z}(x, z)\right| \leq c_{1}\left(|z|+|z|^{p-1}\right), \quad \forall(x, z) \in \mathbb{R}^{N} \times \mathbb{R}^{2} .
$$

Consequently, by the conditions $\left(H_{0}\right)$ and (5), it is easy to see that $\left(H_{4}\right)$ and $\left(H_{5}\right)$ hold. Furthermore, similar to the proof of Lemma 2.2(i) in [23], the condition $\left(\mathrm{H}_{6}\right)$ can be obtained by $\left(H_{0}\right)$ and (5). Indeed, since $p \in\left(2,2^{*}\right)$, we can obtain that $p /(p-2)>\max \{1, N / 2\}$. For some $\tau \in(N / 2, p /(p-2))$, $\tau>1$. If $|z| \geq 1$, then there exists $c^{\prime}>0$ such that

$$
\left|H_{z}(x, z)\right| \leq c^{\prime}|z|^{p-1} .
$$

Choose $r \geq 1$ so large that

$$
\frac{1}{\mu} \leq \frac{1}{2}-\frac{\left(c^{\prime}\right)^{\tau-1}}{|z|^{p-(p-2) \tau}}
$$


whenever $|z| \geq r$. Then, by $\left(H_{0}\right),(6)$, and (7), we obtain

$$
\begin{aligned}
H(x, z) & \leq \frac{1}{\mu} H_{z}(x, z) z \\
& \leq\left(\frac{1}{2}-\frac{\left(c^{\prime}\right)^{\tau-1}}{|z|^{p-(p-2) \tau}}\right) H_{z}(x, z) z \\
& \leq\left(\frac{1}{2}-\frac{\left|H_{z}(x, z)\right|^{\tau-1}}{|z|^{\tau+1}}\right) H_{z}(x, z) z .
\end{aligned}
$$

It follows that

$$
\frac{\left|H_{z}(x, z)\right|^{\tau}}{|z|^{\tau}} \leq \frac{1}{2} H_{z}(x, z) z-H(x, z)=\widetilde{H}(x, z) .
$$

Third, the condition $\left(H_{3}\right)$ is weaker than the condition $\left(H_{1}\right)$ and the condition $\left(\mathrm{H}_{7}\right)$ is weaker than the condition $\left(\mathrm{H}_{2}\right)$. Finally, summing up the above discussion, Theorem $\mathrm{A}$ is the special case of Theorem 1 corresponding to $b(x)=0$.

Throughout this paper, we always assume that $C$ denote any positive constant and may be different in different places. For $0 \neq a=\left(a_{1}, \ldots, a_{N}\right) \in \mathbb{Z}^{N}$, we define $a * z(x):=z\left(x_{1}+\right.$ $\left.a_{1}, \ldots, x_{N}+a_{N}\right)$, where $z=(u, v): \mathbb{R}^{N} \rightarrow \mathbb{R}^{1} \times \mathbb{R}^{1}$.

\section{Variational Setting}

In this section, we will establish variational framework for the system (HES). For the convenience of notation, let $|\cdot|_{p}$ denote the usual $L^{p}$-norm and $(\cdot, \cdot)_{2}$ be the usual $L^{2}$-inner product. Let $X$ and $Y$ be two Banach spaces with norms $\|\cdot\|_{X}$ and $\|\cdot\|_{Y}$; we always choose the equivalent norm $\|(x, y)\|_{X \times Y}=\left(\|x\|_{X}^{2}+\right.$ $\left.\|y\|_{Y}^{2}\right)^{1 / 2}$ on the product space $X \times Y$. In particular, if $X$ and $Y$ are two Hilbert spaces with inner products $(\cdot, \cdot)_{X}$ and $(\cdot, \cdot)_{Y}$, we choose the inner product $((x, y),(w, z))_{X \times Y}=(x, w)_{X}+$ $(y, z)_{Y}$ on the product space $X \times Y$. In order to continue the discussion, we need the following notations. Set

$$
\begin{gathered}
\mathscr{J}=\left(\begin{array}{cc}
0 & -1 \\
1 & 0
\end{array}\right), \\
\mathscr{J}_{0}=\left(\begin{array}{ll}
0 & 1 \\
1 & 0
\end{array}\right),
\end{gathered}
$$

and $\nabla \mathscr{J}=\left(\begin{array}{cc}0 & -\nabla \\ \nabla & 0\end{array}\right)$ is a matrix operator. Let $\mathcal{S}=-\Delta+V$ denote the Schrödinger operator. Denote $A_{0}:=\mathcal{S}_{\mathscr{F}_{0}}$ and

$$
\begin{aligned}
A & :=A_{0}+b \cdot \nabla \mathscr{J} \\
& =\left(\begin{array}{cc}
0 & -\Delta-b \cdot \nabla+V \\
-\Delta+b \cdot \nabla+V & 0
\end{array}\right) .
\end{aligned}
$$

Then (HES) can be rewritten as

$$
A z=H_{z}(x, z), \quad z \in H^{1}\left(\mathbb{R}^{N}, \mathbb{R}^{2}\right) .
$$

Denote by $\sigma(A)$ and $\sigma_{\text {ess }}(A)$ the spectrum and the essential spectrum of the operator $A$, respectively. Set $\mu:=$ $\inf \{\sigma(A) \cap(0, \infty)\}$; then we have the following lemmas.
Lemma 3 (see $\left[19\right.$, Lemma 2.1]). Suppose that $\left(B_{0}\right)$ and $\left(V_{1}\right)$ are satisfied. Then the operator $A$ is a self-adjoint operator on $L^{2}\left(\mathbb{R}^{N}, \mathbb{R}^{2}\right)$ with domain $\mathscr{D}(A)=H^{2}\left(\mathbb{R}^{N}, \mathbb{R}^{2}\right)$.

Lemma 4 (see [19, Lemma 2.3]). Let $\left(B_{0}\right),\left(B_{1}\right)$, and $\left(V_{1}\right)$ be satisfied. Then

(1) $\sigma(A)=\sigma_{\text {ess }}(A)$; that is, $A$ has only essential spectrum;

(2) $\sigma(A) \subset \mathbb{R} \backslash\left(-V_{0}, V_{0}\right)$ and $\sigma(A)$ is symmetric with respect to origin

(3) $V_{0} \leq \mu \leq \max V$.

It follows from Lemmas 3 and 4 that the space $L^{2}\left(\mathbb{R}^{N}, \mathbb{R}^{2}\right)$ possesses the orthogonal decomposition

$$
\begin{gathered}
L^{2}=L^{-} \oplus L^{+}, \\
z=z^{-}+z^{+},
\end{gathered}
$$

such that $A$ is negative definite (resp., positive definite) in $L^{-}$ (resp. $L^{+}$). Let $|A|$ denote the absolute value of $A$ and $|A|^{1 / 2}$ be the square root of $|A|$. Let $E=\mathscr{D}\left(|A|^{1 / 2}\right)$ be the Hilbert space with the inner product

$$
(z, w)=\left(|A|^{1 / 2} z,|A|^{1 / 2} w\right)_{L^{2}},
$$

and norm $\|z\|=(z, z)^{1 / 2}$. E possesses an induced decomposition

$$
\begin{aligned}
E & =E^{-} \oplus E^{+}, \\
E^{ \pm} & =E \cap L^{ \pm},
\end{aligned}
$$

which are orthogonal with respect to the inner products $(\cdot, \cdot)_{2}$ and $(\cdot, \cdot)$ (the above results can be found in [19]).

Lemma 5 (see [19, Lemma 2.4]). $\|\cdot\|$ and $\|\cdot\|_{H^{1}\left(\mathbb{R}^{N}, \mathbb{R}^{2}\right)}$ are equivalent norms. Therefore, $E$ embeds continuously into $L^{p}\left(R^{N}, R^{2}\right)$ for any $p \in\left[2,2^{*}\right]$ and compactly into $L_{\mathrm{loc}}^{p}\left(R^{N}, R^{2}\right)$ for $p \in\left[2,2^{*}\right)$, and there exists constant $a_{p}$ such that

$$
|z|_{p} \leq a_{p}\|z\|, \quad \forall z \in E, p \in\left[2,2^{*}\right] .
$$

On $E$ we define the following functional:

$$
\Phi(z)=\frac{1}{2}\left(\left\|z^{+}\right\|^{2}-\left\|z^{-}\right\|^{2}\right)-\Psi(z),
$$

where $\Psi(z)=\int_{\mathbb{R}^{N}} H(x, z) d x$. It follows from $\left(\mathrm{H}_{3}\right)$ that, for any $\varepsilon>0$, there is $C(\varepsilon)>0$ such that

$$
\begin{gathered}
\left|H_{z}(x, z)\right| \leq \varepsilon|z|+C(\varepsilon)|z|^{p-1}, \\
|H(x, z)| \leq \frac{\varepsilon}{2}|z|^{2}+\frac{C(\varepsilon)}{p}|z|^{p},
\end{gathered}
$$

for all $(x, z)$. Thus, Lemma 5 implies that $\Phi$ is well defined on E. Lemma 4 implies that $\Phi$ is strongly indefinite; such type functional appeared extensively when one considers differential equations via critical point theory; see, for example, [24-27] and the references therein. Our hypotheses imply that $\Phi \in$ $C^{1}(E, \mathbb{R})$ (see Lemma 3.10 in [27]) and a standard argument shows that the critical points of $\Phi$ are solutions of (HES). 


\section{The Abstract Critical Point Theorem}

In order to study the critical points of $\Phi$, we now recall a abstract critical point theorem developed recently in $[10,20]$. Let $E$ be a Banach space with direct sum $E=X \oplus Y$ and corresponding projections $P_{X}, P_{Y}$ onto $X, Y$. We assume that the Banach space $X$ is separable and reflexive. Let $\delta \subset X^{*}$ be a dense subset; for each $s \in \mathcal{S}$ there is a seminorm on $E$ defined by

$$
\begin{aligned}
& p_{s}: E \longrightarrow R, \\
& p_{s}(z)=|s(x)|+\|y\| \quad \text { for } z=x+y \in E .
\end{aligned}
$$

Denote by $\mathscr{T}_{\delta}$ and $\mathscr{T}_{w}$ the topology induced by seminorm family $\left\{p_{s}\right\}$ and the weak-topology on $E$, respectively. $\mathscr{T}_{w^{*}}$ denotes the weak ${ }^{*}$-topology on $E^{*}$. Now, some notations and definitions are needed.

For a functional $\Phi \in C^{1}(E, \mathbb{R})$, we write $\Phi_{a}=\{z \in E \mid$ $\Phi(z) \geq a\}, \Phi^{b}=\{z \in E \mid \Phi(z) \leq b\}$, and $\Phi_{a}^{b}=\Phi_{a} \cap \Phi^{b}$.

Suppose

$\left(\Phi_{0}\right)$ for any $c \in \mathbb{R}, \Phi_{c}$ is $\mathscr{T}_{\mathcal{\delta}}$-closed, and $\Phi^{\prime}:\left(\Phi_{c}, \mathscr{T}_{\delta}\right) \rightarrow$ $\left(E^{*}, \mathscr{T}_{w^{*}}\right)$ is continuous;

$\left(\Phi_{1}\right)$ for any $c>0$, there exists $\xi>0$ such that $\|z\|<\xi\left\|P_{Y} z\right\|$ for all $z \in \Phi_{c}$;

$\left(\Phi_{2}\right)$ there exists $\rho>0$ such that $\kappa:=\inf \Phi\left(S_{\rho} \cap Y\right)>0$, where $S_{\rho}:=\{z \in E:\|z\|=\rho\}$.

Theorem 6 (see [10] or [20]). Let $\left(\Phi_{0}\right)-\left(\Phi_{2}\right)$ be satisfied and suppose there are $R>\rho>0$ and $e \in Y$ with $\|e\|=1$ such that $\sup \Phi(\partial Q) \leq \kappa$ where $Q:=\{z=x+$ te $: x \in X, t \geq 0,\|z\| \leq$ $R\}$. Then, $\Phi$ has a $\left(C_{c}\right)$-sequence with $\kappa \leq c \leq \bar{c}:=\sup \Phi(Q)$. Moreover, if $\Phi$ satisfies the $(C)_{c}$-condition for all $c \leq \bar{c}$ then $\Phi$ has a critical point $z$ with $\kappa \leq \Phi(z) \leq \bar{c}$.

Lemma 7 (see [10] or [20]). Let

$$
\Phi(z)=\frac{1}{2}\left(\|y\|^{2}-\|x\|^{2}\right)-\Psi(z),
$$

$$
\text { for } z=x+y \in E=X \oplus Y .
$$

Suppose

(1) $\Psi \in C^{1}(E, \mathbb{R})$ is bounded from below;

(2) $\Psi:\left(E, \mathscr{T}_{w}\right) \rightarrow \mathbb{R}$ is sequentially lower semicontinuous; that is, $z_{n} \rightarrow z$ in $E$ implies $\Psi(z) \leq$ $\liminf _{n \rightarrow \infty} \Psi\left(z_{n}\right)$;

(3) $\Psi^{\prime}:\left(E, \mathscr{T}_{w}\right) \rightarrow\left(E^{*}, \mathscr{T}_{w^{*}}\right)$ is sequentially continuous;

(4) $v: E \rightarrow R, v(z)=\|z\|^{2}$ is $C^{1}$ and $v^{\prime}:\left(E, \mathscr{T}_{w}\right) \rightarrow$ $\left(E^{*}, \mathscr{T}_{w^{*}}\right)$ is sequentially continuous.

Then $\Phi$ satisfies $\Phi_{0}$.

\section{The Limit Equation}

In this section, we study the following limit equation related to (HES),

$$
A z=h_{\infty}(|z|) z=H_{\infty}^{\prime}(z),
$$

$(\mathrm{HES})_{\infty}$ where $h_{\infty}$ is given in $\left(H_{2}\right), H_{\infty}(z)=\int_{0}^{|z|} h_{\infty}(s) s d s$, and $H_{\infty}^{\prime}(z)=\nabla_{z} H_{\infty}(z)=\left(\nabla_{u} H_{\infty}(z), \nabla_{v} H_{\infty}(z)\right)$. By virtue of $\left(H_{3}\right)-\left(H_{7}\right)$, we have firstly the following lemma.

Lemma 8. $h_{\infty}$ and $H_{\infty}$ possess the following properties.

(i) $h_{\infty}(|z|)=o(1)$ as $|z| \rightarrow 0$ and $h_{\infty}(|z|) \leq c_{1}\left(1+|z|^{p-2}\right)$ for some $p \in\left(2,2^{*}\right)$.

(ii) $H_{\infty}(z) /|z|^{2} \rightarrow+\infty$ as $|z| \rightarrow+\infty$.

(iii) $\widetilde{H}_{\infty}(z)>0$ whenever $z \neq 0$, where $\widetilde{H}_{\infty}(z)=(1 /$ 2) $H_{\infty}^{\prime}(z) z-H_{\infty}(z)$.

(iv) $\left|H_{\infty}^{\prime}(z)\right|^{\tau} \leq c_{2} \widetilde{H}_{\infty}(z)|z|^{\tau}$ whenever $|z|$ large enough.

(v) $\widetilde{H}_{\infty}(z) \rightarrow+\infty$ as $|z| \rightarrow+\infty$.

Proof. (i) It is clear by $\left(\mathrm{H}_{3}\right)$ and $\left(\mathrm{H}_{7}\right)$.

(ii) By $\left(H_{4}\right)$, for any $M>0$, there is $R_{M}>0$ such that $H(x, z) /|z|^{2} \geq M$ whenever $|z| \geq R_{M}$. Hence

$$
\begin{aligned}
\frac{H_{\infty}(z)}{|z|^{2}} & =\frac{H_{\infty}(z)-H(x, z)}{|z|^{2}}+\frac{H(x, z)}{|z|^{2}} \\
& \geq \frac{H_{\infty}(z)-H(x, z)}{|z|^{2}}+M,
\end{aligned}
$$

for all $|z| \geq R_{M}$. Observe that

$$
\begin{aligned}
0 & \leq H(x, z)-H_{\infty}(z) \\
& =\int_{0}^{1}\left(H_{z}(x, \theta z)-H_{\infty}^{\prime}(\theta z)\right) z d \theta ;
\end{aligned}
$$

it follows from $\left(H_{7}\right)$ that $H(x, z) \rightarrow H_{\infty}(z)$ as $|x| \rightarrow+\infty$. Letting $|x| \rightarrow+\infty$ we get $H_{\infty}(z) /|z|^{2} \geq M$ for all $|z| \geq R_{M}$.

(iii) Since $H(x, z) \rightarrow H_{\infty}(z)$ as $|x| \rightarrow+\infty$, it follows from $\left(H_{5}\right)$ that

$$
\widetilde{H}_{\infty}(z)=\lim _{|x| \rightarrow+\infty} \widetilde{H}(x, z)>0, \quad \text { if } z \neq 0 .
$$

(iv) By $\left(H_{6}\right)$, for $|z|$ large enough

$$
\begin{aligned}
\frac{\left|H_{\infty}^{\prime}(z)\right|}{|z|} & \leq \frac{\left|H_{\infty}^{\prime}(z)-H_{z}(x, z)\right|}{|z|}+\frac{\left|H_{z}(x, z)\right|}{|z|} \\
& \leq \frac{\left|H_{\infty}^{\prime}(z)-H_{z}(x, z)\right|}{|z|}+c_{2}^{1 / \tau} \widetilde{H}(x, z)^{1 / \tau} .
\end{aligned}
$$

Letting $|x| \rightarrow+\infty$, we obtain

$$
\frac{\left|H_{\infty}^{\prime}(z)\right|}{|z|} \leq c_{2}^{1 / \tau} \widetilde{H}_{\infty}(z)^{1 / \tau} .
$$

(v) It follows from (ii)-(iv) that

$$
c_{2} \widetilde{H}_{\infty}(z) \geq\left(\frac{\left|H_{\infty}^{\prime}(z)\right|}{|z|}\right)^{\tau} \geq\left(\frac{2 H_{\infty}(z)}{|z|^{2}}\right)^{\tau} \longrightarrow+\infty,
$$

as $|z| \rightarrow+\infty$. 
Now, we set

$$
\Psi_{\infty}(z)=\int_{R^{N}} H_{\infty}(z) d x
$$

and define the functional

$$
\begin{aligned}
& F(z)=\frac{1}{2}\left(\left\|z^{+}\right\|^{2}-\left\|z^{-}\right\|^{2}\right)-\Psi_{\infty}(z), \\
& \forall z=z^{-}+z^{+} \in E .
\end{aligned}
$$

By Lemma 8 , for any $\varepsilon>0$, there is $C(\varepsilon)>0$ such that

$$
\begin{aligned}
& \left|H_{\infty}^{\prime}(z)\right| \leq \varepsilon|z|+C(\varepsilon)|z|^{p-1}, \quad \forall(x, z) \in R^{N} \times R^{2} \\
& \left|H_{\infty}(z)\right| \leq \frac{\varepsilon}{2}|z|^{2}+\frac{C(\varepsilon)}{p}|z|^{p}, \quad \forall(x, z) \in R^{N} \times R^{2} .
\end{aligned}
$$

It follows from (30) and (31) that $F \in C^{1}(E, \mathbb{R})$ is well defined and its critical points are solutions of $(\mathrm{HES})_{\infty}$.

Lemma 9. F possesses the following properties:

(1) $\Psi_{\infty}$ is weakly sequentially lower semicontinuous and $F^{\prime}$ is weak sequentially continuous.

(2) There is $\rho>0$ such that $\kappa:=\inf F\left(\partial B_{\rho} \cap E^{+}\right)>0$, where $\partial B_{\rho}:=\{z \in E:\|z\|=\rho\}$.

Proof. (1) Suppose $z_{n} \rightarrow z$ in $E$. Going if necessary to a subsequence, we can assume $z_{n} \rightarrow z$ in $L_{\text {loc }}^{p}\left(\mathbb{R}^{N}, \mathbb{R}^{2}\right)$ for $p \in\left[2,2^{*}\right)$ and $z_{n}(x) \rightarrow z(x)$ a.e. in $\mathbb{R}^{N}$. Hence $H_{\infty}\left(z_{n}(x)\right) \rightarrow$ $H_{\infty}(z(x))$ a.e. in $\mathbb{R}^{N}$. Thus

$$
\begin{aligned}
\Psi_{\infty}(z) & =\int_{\mathbb{R}^{N}} H_{\infty}(z) d x=\int_{\mathbb{R}^{N}} \lim _{n \rightarrow \infty} H_{\infty}\left(z_{n}\right) d x \\
& \leq \liminf _{n \rightarrow \infty} \int_{\mathbb{R}^{N}} H_{\infty}\left(z_{n}\right) d x \\
& =\liminf _{n \rightarrow \infty} \Psi_{\infty}\left(z_{n}\right) .
\end{aligned}
$$

Next it is sufficient to show that $\Psi_{\infty}^{\prime}$ is weak sequentially continuous. Indeed, by (30) and $z_{n} \rightarrow z$ in $L_{\text {loc }}^{p}\left(\mathbb{R}^{N}, \mathbb{R}^{2}\right)$, $2 \leq p<2^{*}$, it follows from Theorem A.2 in [27] that

$$
H_{\infty}^{\prime}\left(z_{n}\right) \longrightarrow H_{\infty}^{\prime}(z), \quad \text { in } L_{\text {loc }}^{p / p-1}\left(\mathbb{R}^{N}, \mathbb{R}^{2}\right)
$$

Furthermore, for each fixed $\varphi \in E$, one has that, for any $\varepsilon_{1}>$ 0 , there exists $r_{0}>0$ such that

$$
\left(\int_{\mathbb{R}^{N} \backslash B_{r_{0}}(0)}|\varphi(x)|^{p} d x\right)^{1 / p}<\varepsilon_{1} .
$$

Hence, for large $n$, it follows from (30), (33), (34), and Hölder inequality that

$$
\begin{aligned}
& \left|\int_{\mathbb{R}^{N}}\left(H_{\infty}^{\prime}\left(z_{n}\right)-H_{\infty}^{\prime}(z)\right) \varphi d x\right| \leq \int_{\mathbb{R}^{N}} \mid H_{\infty}^{\prime}\left(z_{n}\right) \\
& -H_{\infty}^{\prime}(z)|| \varphi \mid d x \leq\left(\int_{B_{r_{0}}(0)} \mid H_{\infty}^{\prime}\left(z_{n}\right)\right. \\
& \left.\quad-\left.H_{\infty}^{\prime}(z)\right|^{p /(p-1)} d x\right)^{(p-1) / p} \\
& \quad \cdot\left(\int_{B_{r_{0}}(0)}|\varphi|^{p} d x\right)^{1 / p}+\int_{\mathbb{R}^{N} \backslash B_{r_{0}}(0)}\left[\varepsilon\left(\left|z_{n}\right|+|z|\right)\right. \\
& \left.\quad+C(\varepsilon)\left(\left|z_{n}\right|^{p-1}+|z|^{p-1}\right)\right]|\varphi| d x \\
& \quad \leq\left(\int_{B_{r_{0}}(0)}\left|H_{\infty}^{\prime}\left(z_{n}\right)-H_{\infty}^{\prime}(z)\right|^{p /(p-1)} d x\right)^{(p-1) / p} \\
& \quad+\left(\int_{B_{r_{0}}(0)}|\varphi|^{p} d x\right)^{1 / p} \\
& +\varepsilon\left(\int_{\mathbb{R}^{N} \backslash B_{r_{0}}(0)}|\varphi(x)|^{2} d x\right)^{1 / 2}\left(\left|z_{n}\right|_{2}+|z|_{2}\right) \\
& +\varepsilon_{1} C(\varepsilon)\left(\left|z_{n}\right|_{p}^{p-1}+|z|_{p}^{p-1}\right) .
\end{aligned}
$$

Therefore,

$$
\begin{aligned}
& \left|\left\langle\Psi_{\infty}^{\prime}\left(z_{n}\right), \varphi\right\rangle-\left\langle\Psi_{\infty}^{\prime}(z), \varphi\right\rangle\right| \\
& =\left|\int_{\mathbb{R}^{N}}\left(H_{\infty}^{\prime}\left(z_{n}\right)-H_{\infty}^{\prime}(z)\right) \varphi d x\right| \longrightarrow 0, \\
& \text { as } n \longrightarrow \infty \\
& \text { (2) For any } z^{+} \in E^{+} \text {, it follows from (31) that } \\
& F\left(z^{+}\right)=\frac{1}{2}\left\|z^{+}\right\|^{2}-\int_{R^{N}} H_{\infty}\left(z^{+}\right) d x \\
& \geq \frac{1}{2}\left\|z^{+}\right\|^{2}-\int_{R^{N}}\left(\frac{\varepsilon}{2}\left|z^{+}\right|^{2}+\frac{C(\varepsilon)}{p}\left|z^{+}\right|^{p}\right) d x \\
& \geq\left(\frac{1}{2}-C \varepsilon\right)\left\|z^{+}\right\|^{2}-C\left\|z^{+}\right\|^{p} .
\end{aligned}
$$

The conclusion follows because $p>2$.

Now, we choose a number $\gamma>0$ such that $\max _{x \in R^{N}} V(x)<\gamma$. From Lemma 8(ii), there is $r>0$ such that $H_{\infty}(z) \geq \gamma|z|^{2}$ whenever $|z| \geq r$. Let $\left\{E_{\lambda}\right\}_{\lambda \in R}$ be the spectrum family of the operator $A$. It follows from Lemma 4 that $\left(E_{\gamma}-F_{0}\right) L^{2}$ is a infinite dimension subspace of $E^{+}$and

$$
V_{0}|z|_{2}^{2} \leq \underline{\mu}|z|_{2}^{2} \leq\|z\|^{2} \leq \gamma|z|_{2}^{2}, \quad \forall z \in\left(E_{\gamma}-E_{0}\right) L^{2} .
$$

We have the following result. 
Lemma 10. For any finite dimensional subspace $E_{W}$ of $\left(E_{\gamma}-\right.$ $\left.E_{0}\right) L^{2}$,

$$
F(z) \longrightarrow-\infty, \quad \text { as }\|z\| \longrightarrow \infty, z \in E^{-} \oplus E_{W} .
$$

Proof. If not, then there are $M>0$ and $\left\{z_{n}\right\} \subset E^{-} \oplus E_{W}$ with $\left\|z_{n}\right\| \rightarrow \infty$ such that $F\left(z_{n}\right) \geq-M$ for all $n$. Denote $y_{n}:=$ $z_{n} /\left\|z_{n}\right\|$, passing to a subsequence if necessary; we can assume that $y_{n} \rightarrow y, y_{n}^{-} \rightarrow y^{-}$, and $y_{n}^{+} \rightarrow y^{+}$. Then

$$
\begin{aligned}
\frac{1}{2}\left(\left\|y_{n}^{+}\right\|^{2}-\left\|y_{n}^{-}\right\|^{2}\right) \geq & \frac{1}{2}\left(\left\|y_{n}^{+}\right\|^{2}-\left\|y_{n}^{-}\right\|^{2}\right) \\
& -\int_{R^{N}} \frac{H_{\infty}\left(z_{n}\right)}{\left\|z_{n}\right\|^{2}} d x=\frac{F\left(z_{n}\right)}{\left\|z_{n}\right\|^{2}} \\
\geq & \frac{-M}{\left\|z_{n}\right\|^{2}},
\end{aligned}
$$

which yields that

$$
\frac{1}{2}\left\|y_{n}^{-}\right\|^{2} \leq \frac{1}{2}\left\|y_{n}^{+}\right\|^{2}+\frac{M}{\left\|z_{n}\right\|^{2}} .
$$

We claim that $y^{+} \neq 0$. Indeed, if not then it follows from (41) that $\left\|y_{n}^{-}\right\| \rightarrow 0$. Thus $\left\|y_{n}\right\| \rightarrow 0$, which contradicts with $\left\|y_{n}\right\|=$ 1. By (38), we get

$$
\begin{aligned}
& \left\|y^{+}\right\|^{2}-\left\|y^{-}\right\|^{2}-2 \gamma|y|_{2}^{2} \\
& \quad \leq \gamma\left|y^{+}\right|_{2}^{2}-\left\|y^{-}\right\|^{2}-2 \gamma\left|y^{+}\right|_{2}^{2}-2 \gamma\left|y^{-}\right|_{2}^{2} \\
& \leq-\gamma\left|y^{+}\right|_{2}^{2}-\left\|y^{-}\right\|^{2}-2 \gamma\left|y^{-}\right|_{2}^{2}<0 .
\end{aligned}
$$

Hence, there exists $R>0$ such that

$$
\left\|y^{+}\right\|^{2}-\left\|y^{-}\right\|^{2}-2 \gamma \int_{B_{R}}|y|^{2} d x<0,
$$

where $B_{R}:=\left\{x \in R^{N}:|x| \leq R\right\}$. Note that

$$
\begin{aligned}
\frac{F\left(z_{n}\right)}{\left\|z_{n}\right\|^{2} \leq} & \frac{1}{2}\left(\left\|y_{n}^{+}\right\|^{2}-\left\|y_{n}^{-}\right\|^{2}\right)-\int_{B_{R}} \frac{H_{\infty}\left(z_{n}\right)}{\left\|z_{n}\right\|^{2}} d x \\
= & \frac{1}{2}\left(\left\|y_{n}^{+}\right\|^{2}-\left\|y_{n}^{-}\right\|^{2}-2 \gamma \int_{B_{R}}\left|y_{n}\right|^{2} d x\right) \\
& -\int_{B_{R}} \frac{H_{\infty}\left(z_{n}\right)-\gamma\left|z_{n}\right|^{2}}{\left\|z_{n}\right\|^{2}} d x \\
\leq & \frac{1}{2}\left(\left\|y_{n}^{+}\right\|^{2}-\left\|y_{n}^{-}\right\|^{2}-2 \gamma \int_{B_{R}}\left|y_{n}\right|^{2} d x\right) \\
& -\int_{B_{R} \cap\left\{x \in R^{N}:\left|z_{n}\right| \leq r\right\}} \frac{H_{\infty}\left(z_{n}\right)-\gamma\left|z_{n}\right|^{2}}{\left\|z_{n}\right\|^{2}} d x \\
\leq & \frac{1}{2}\left(\left\|y_{n}^{+}\right\|^{2}-\left\|y_{n}^{-}\right\|^{2}-2 \gamma \int_{B_{R}}\left|y_{n}\right|^{2} d x\right) \\
& +\frac{\gamma r^{2}\left|B_{R}\right|}{\left\|z_{n}\right\|^{2}} .
\end{aligned}
$$

Hence

$$
\begin{aligned}
0 & \leq \lim _{n \rightarrow \infty}\left[\frac{1}{2}\left(\left\|y_{n}^{+}\right\|^{2}-\left\|y_{n}^{-}\right\|^{2}\right)-\int_{B_{R}} \frac{H_{\infty}\left(z_{n}\right)}{\left\|z_{n}\right\|^{2}} d x\right] \\
& \leq \frac{1}{2}\left(\left\|y^{+}\right\|^{2}-\left\|y^{-}\right\|^{2}-2 \gamma \int_{B_{R}}|y|^{2} d x\right)<0 .
\end{aligned}
$$

Now the desired conclusion follows from this contradiction.

As a consequence, we have the following.

Lemma 11. Let $\kappa>0$ be given by Lemma 9. Then, letting e $\epsilon$ $E_{W}$ with $\|e\|=1$, there is $R_{1}>0$ such that $\left.F\right|_{\partial Q} \leq \kappa$, where $Q:=\left\{z=z^{-}+s e: z^{-} \in E^{-}, s \geq 0,\|z\| \leq R_{1}\right\}$.

Lemma 12. Let $\left\{z_{n}\right\}$ be any $(C)_{c}$-sequence for $F$. That is, $\left\{z_{n}\right\} \subset$ $E$ is such that

$$
\begin{aligned}
F\left(z_{n}\right) & \longrightarrow c, \\
\left(1+\left\|z_{n}\right\|\right) F^{\prime}\left(z_{n}\right) & \longrightarrow 0,
\end{aligned}
$$

as $n \rightarrow \infty$. Then it is bounded and $c \geq 0$. Moreover, there is a subsequence still denoted by $\left\{z_{n}\right\}$ satisfying $z_{n} \rightarrow z, F\left(z_{n}-\right.$ $z) \rightarrow c-F(z)$ and $F^{\prime}\left(z_{n}-z\right) \rightarrow 0$, as $n \rightarrow \infty$.

Proof. Let $\left\{z_{n}\right\} \subset E$ be such that

$$
\begin{aligned}
F\left(z_{n}\right) & \longrightarrow c, \\
\left(1+\left\|z_{n}\right\|\right) F^{\prime}\left(z_{n}\right) & \longrightarrow 0 .
\end{aligned}
$$

Then, for large $n$, one has

$$
\begin{aligned}
c+o(1) & \geq F\left(z_{n}\right)-\frac{1}{2}\left\langle F^{\prime}\left(z_{n}\right), z_{n}\right\rangle \\
& =\int_{\mathbb{R}^{N}}\left[\frac{1}{2} h_{\infty}\left(\left|z_{n}\right|\right)\left|z_{n}\right|^{2}-H_{\infty}\left(z_{n}\right)\right] d x \\
& =\int_{\mathbb{R}^{N}} \widetilde{H}_{\infty}\left(z_{n}\right) d x \geq 0,
\end{aligned}
$$

which implies $c \geq 0$. If $\left\{z_{n}\right\}$ is unbounded in $E$, up to a subsequence if necessary, we can assume that $\left\|z_{n}\right\| \rightarrow+\infty$. Set $w_{n}=z_{n} /\left\|z_{n}\right\|$. Then $\left\|w_{n}\right\|=1$ and $\left|w_{n}\right|_{p} \leq a_{p}\left\|w_{n}\right\|=a_{p}$ for each $2 \leq p \leq 2^{*}$. Note that

$$
\begin{aligned}
& \frac{\left\langle F^{\prime}\left(z_{n}\right), z_{n}^{+}-z_{n}^{-}\right\rangle}{\left\|z_{n}\right\|^{2}} \\
& =1-\int_{\mathbb{R}^{N}} \frac{h_{\infty}\left(\left|z_{n}\right|\right) z_{n}\left(z_{n}^{+}-z_{n}^{-}\right)}{\left\|z_{n}\right\|^{2}} d x .
\end{aligned}
$$

Hence, one has

$$
\begin{aligned}
& \lim _{n \rightarrow \infty} \int_{\mathbb{R}^{N}} \frac{h_{\infty}\left(\left|z_{n}\right|\right) z_{n}\left(z_{n}^{+}-z_{n}^{-}\right)}{\left\|z_{n}\right\|^{2}} d x \\
& \quad=\lim _{n \rightarrow \infty} \int_{\mathbb{R}^{N}} h_{\infty}\left(\left|z_{n}\right|\right) w_{n}\left(w_{n}^{+}-w_{n}^{-}\right) d x=1 .
\end{aligned}
$$


On the other hand, for $r \geq 0$ and $0 \leq \alpha<\beta \leq+\infty$, set

$$
\begin{aligned}
g(r) & :=\inf \left\{\widetilde{H}_{\infty}(z): z \in \mathbb{R}^{2},|z| \geq r\right\}, \\
O_{n}(\alpha, \beta) & :=\left\{x \in \mathbb{R}^{N}: \alpha \leq\left|z_{n}(x)\right|<\beta\right\}, \\
S_{\alpha}^{\beta} & :=\inf \left\{\frac{\widetilde{H}_{\infty}(z)}{|z|^{2}}: z \in \mathbb{R}^{2}, \alpha \leq|z|<\beta\right\} .
\end{aligned}
$$

Then, by Lemma 8, we have $g(r)>0$ for all $r>0$ and $g(r) \rightarrow$ $+\infty$ as $r \rightarrow+\infty$. For large $\alpha>0$, one has $S_{\alpha}^{\beta}>0$ and

$$
\widetilde{H}_{\infty}\left(z_{n}(x)\right) \geq S_{\alpha}^{\beta}\left|z_{n}(x)\right|^{2}, \quad \forall x \in O_{n}(\alpha, \beta)
$$

Consequently, for large $n$ and $\alpha$, whenever $\alpha<\beta$ one has

$$
\begin{aligned}
c+o(1) \geq & \int_{\mathrm{O}_{n}(0, \alpha)} \widetilde{H}_{\infty}\left(z_{n}\right) d x+\int_{\mathrm{O}_{n}(\alpha, \beta)} \widetilde{H}_{\infty}\left(z_{n}\right) d x \\
& +\int_{\mathrm{O}_{n}(\beta,+\infty)} \widetilde{H}_{\infty}\left(z_{n}\right) d x \\
\geq & \int_{O_{n}(0, \alpha)} \widetilde{H}_{\infty}\left(z_{n}\right) d x+S_{\alpha}^{\beta} \int_{O_{n}(\alpha, \beta)}\left|z_{n}\right|^{2} d x \\
& +g(\beta)\left|O_{n}(\beta,+\infty)\right| .
\end{aligned}
$$

Since $\widetilde{H}_{\infty}\left(z_{n}\right) \geq 0, \lim _{\beta \rightarrow+\infty} g(\beta)=+\infty$ implies

$$
\lim _{\beta \rightarrow+\infty}\left|O_{n}(\beta,+\infty)\right|=0, \quad \text { uniformly in } n \text {. }
$$

For any $s \in\left(2,2^{*}\right)$, we choose $r \in\left(s, 2^{*}\right)$. Using the Hölder inequality we have

$$
\begin{aligned}
& \int_{O_{n}(\beta,+\infty)}\left|w_{n}\right|^{s} d x \\
& \quad \leq\left|O_{n}(\beta,+\infty)\right|^{(r-s) / r}\left(\int_{R^{N}}\left|w_{n}\right|^{r} d x\right)^{s / r} \\
& \quad \leq a_{r}^{s}\left|O_{n}(\beta,+\infty)\right|^{(r-s) / r} \longrightarrow 0
\end{aligned}
$$

as $\beta \rightarrow+\infty$ uniformly in $n$ and

$$
\begin{aligned}
\int_{O_{n}(\alpha, \beta)}\left|w_{n}\right|^{2} d x & \leq \frac{1}{\left\|z_{n}\right\|^{2}} \int_{O_{n}(\alpha, \beta)}\left|z_{n}\right|^{2} d x \leq \frac{C}{S_{\alpha}^{\beta}\left\|z_{n}\right\|^{2}} \\
& \longrightarrow 0
\end{aligned}
$$

as $n \rightarrow \infty$. Therefore,

$$
\begin{gathered}
\int_{O_{n}(\alpha, \beta)}\left|w_{n}\right|^{s} d x \leq\left(\int_{O_{n}(\alpha, \beta)}\left|w_{n}\right|^{r} d x\right)^{(s-2) /(r-2)} \\
\cdot\left(\int_{O_{n}(\alpha, \beta)}\left|w_{n}\right|^{2} d x\right)^{(r-s) /(r-2)} \\
\leq C\left(\int_{O_{n}(\alpha, \beta)}\left|w_{n}\right|^{2} d x\right)^{(r-s) /(r-2)} \longrightarrow 0
\end{gathered}
$$

as $n \rightarrow \infty$. Let $0<\varepsilon<1 / 3$ be given. It follows from Lemma 8 that there is $\alpha(\varepsilon)$ such that $\left|h_{\infty}(|z|)\right|<\varepsilon / a_{2}^{2}$ for all $|z| \leq \alpha(\varepsilon)$. Consequently, we have

$$
\int_{O_{n}(0, \alpha(\varepsilon))} h_{\infty}\left(\left|z_{n}\right|\right) w_{n}\left(w_{n}^{+}-w_{n}^{-}\right) d x \leq \frac{\varepsilon}{a_{2}^{2}}\left|w_{n}\right|_{2}^{2} \leq \varepsilon,
$$

for all $n$. By Lemma 8 , we define $\mu=2 \tau /(\tau-1)$ and $\mu^{\prime}=\mu / 2$. By (55), we can take $\beta(\varepsilon)$ so large that

$$
\begin{aligned}
& \left|\int_{O_{n}(\beta(\varepsilon),+\infty)} h_{\infty}\left(\left|z_{n}\right|\right) w_{n}\left(w_{n}^{+}-w_{n}^{-}\right) d x\right| \\
& \quad \leq\left(\int_{O_{n}(\beta(\varepsilon),+\infty)}\left|h_{\infty}\left(\left|z_{n}\right|\right)\right|^{\tau} d x\right)^{1 / \tau} \\
& \cdot\left(\int_{O_{n}(\beta(\varepsilon),+\infty)}\left(\left|w_{n}^{+}-w_{n}^{-}\right|\left|w_{n}\right|\right)^{\mu^{\prime}} d x\right)^{1 / \mu^{\prime}} \\
& \quad \leq\left(\int_{O_{n}(\beta(\varepsilon),+\infty)} c_{2} \widetilde{H}_{\infty}\left(z_{n}\right) d x\right)^{1 / \tau} \\
& \quad \cdot\left(\int_{\mathbb{R}^{N}}\left|w_{n}^{+}-w_{n}^{-}\right|^{\mu} d x\right)^{1 / \mu} \\
& \quad \cdot\left(\int_{O_{n}(\beta(\varepsilon),+\infty)}\left|w_{n}\right|^{\mu} d x\right)^{1 / \mu} \leq \varepsilon
\end{aligned}
$$

for all $n$. For fixed $0<\alpha(\varepsilon)<\beta(\varepsilon)$, it follows from (57) that there is $n_{0}$ such that

$$
\begin{aligned}
& \int_{O_{n}(\alpha(\varepsilon), \beta(\varepsilon))} h_{\infty}\left(\left|z_{n}\right|\right) w_{n}\left(w_{n}^{+}-w_{n}^{-}\right) d x \\
& \quad \leq C \int_{O_{n}(\alpha(\varepsilon), \beta(\varepsilon))}\left|w_{n}\right|^{2} d x \leq \varepsilon, \quad \forall n \geq n_{0} .
\end{aligned}
$$

Now the combination of (58)-(60) implies that for $n \geq n_{0}$

$$
\int_{\mathbb{R}^{N}} h_{\infty}\left(\left|z_{n}\right|\right) w_{n}\left(w_{n}^{+}-w_{n}^{-}\right) d x \leq 3 \varepsilon<1
$$

This contradicts with (50). Hence, $\left\{z_{n}\right\}$ is bounded. Passing to a subsequence if necessary, we can assume that $z_{n} \rightarrow z$ in $E$, $z_{n} \rightarrow z$ in $L_{\text {loc }}^{p}\left(\mathbb{R}^{N}, \mathbb{R}^{2}\right)$ for $p \in\left[2,2^{*}\right)$, and $z_{n}(x) \rightarrow z(x)$ a.e. in $\mathbb{R}^{N}$. Since $(\text { HES })_{\infty}$ is an autonomous system, by $\left(V_{1}\right)$ and Lemma 8, we know that all of the conditions of Lemma 5.7 in [20] are satisfied. Hence we have $F\left(z_{n}-z\right) \rightarrow c-F(z)$ and $F^{\prime}\left(z_{n}-z\right) \rightarrow 0$, as $n \rightarrow \infty$.

Lemma 13. Let $\widehat{\mathscr{K}}:=\left\{z \in E: F^{\prime}(z)=0\right\}$ be the critical set of F. Then $\widehat{\mathscr{K}} \backslash\{0\} \neq \emptyset$ and $\widehat{C}:=\inf \{F(z): z \in \widehat{\mathscr{K}} \backslash\{0\}\}>0$ is attained.

Proof. Set $X=E^{-}$and $Y=E^{+}$. Then $E=X \oplus Y$. For any $c>0$ and $z \in F_{c}$, using the fact that $\Psi_{\infty} \geq 0$ one has

$$
0<c \leq \frac{1}{2}\left(\left\|z^{+}\right\|^{2}-\left\|z^{-}\right\|^{2}\right) .
$$


This yields $\left\|z^{-}\right\|<\left\|z^{+}\right\|$, and hence $\|z\| \leq 2\left\|z^{+}\right\|$. $F$ satisfies $\left(\Phi_{1}\right)$. By virtue of Lemma 7 and conclusion (1) of Lemma 9, it follows that $\left(\Phi_{0}\right)$ holds. From conclusion (2) of Lemma 9, we know that $\left(\Phi_{2}\right)$ holds. Thus, combining with Lemma 11, we know that all of the conditions of Theorem 6 hold. Then there is $(C)_{c}$ sequence $\left\{z_{n}\right\}$ for $F$ with $\kappa \leq c \leq \sup F(Q)$. By Lemma $12,(C)_{c}$ sequence $\left\{z_{n}\right\}$ is bounded in $E$. For the concentration functions $\left|z_{n}\right|^{2}$, there are only two cases needed to be considered: vanishing and nonvanishing. If vanishing occurs, by the vanishing lemma

$$
\begin{aligned}
\int_{\mathbb{R}^{N}} h_{\infty}\left(\left|z_{n}\right|\right)\left|z_{n}\right|^{2} d x & =o(1), \\
\int_{\mathbb{R}^{N}} H_{\infty}\left(z_{n}\right) d x & =o(1) .
\end{aligned}
$$

Hence

$$
\begin{aligned}
o(1) & =\left\langle F^{\prime}\left(z_{n}\right), z_{n}\right\rangle \\
& =\left\|z_{n}^{+}\right\|^{2}-\left\|z_{n}^{-}\right\|^{2}-\int_{\mathbb{R}^{N}} h_{\infty}\left(\left|z_{n}\right|\right)\left|z_{n}\right|^{2} d x,
\end{aligned}
$$

which implies that $\left\|z_{n}^{+}\right\|^{2}-\left\|z_{n}^{-}\right\|^{2}=o(1)$. Hence $F\left(z_{n}\right) \rightarrow 0$ as $n \rightarrow \infty$. This contradicts with $F\left(z_{n}\right) \rightarrow c \geq \kappa>0$ as $n \rightarrow \infty$. So nonvanishing occurs; that is, there exist $\alpha>0, R<\infty$ and $\left\{a_{n}\right\} \subset \mathbb{R}^{N}$ such that

$$
\liminf _{n \rightarrow \infty} \int_{B\left(a_{n}, R\right)}\left|z_{n}\right|^{2} d x \geq \alpha
$$

Choose $g_{n} \in \mathbb{Z}^{N}$ such that $\left|g_{n}-a_{n}\right|=\min \left\{\left|g-a_{n}\right|: g \in \mathbb{Z}^{N}\right\}$. Thus $\left|g_{n}-a_{n}\right| \leq(1 / 2) \sqrt{N}$. Setting $\widetilde{z}_{n}(x)=g_{n} * z_{n}$, by the invariance under translation of $F,\left\{\widetilde{z}_{n}\right\}$ is a $(C)_{c}$-sequence of $F$ and $\widetilde{z}_{n} \rightarrow \widetilde{z}$. From

$$
\liminf _{n \rightarrow \infty} \int_{B(0, R+(1 / 2) \sqrt{N})}\left|\widetilde{z}_{n}\right|^{2} d x \geq \alpha>0
$$

we see that $\widetilde{z} \neq 0$, and hence $\widetilde{z}$ is a nontrivial critical point of $F$. Therefore

$$
\widehat{\mathscr{K}} \backslash\{0\} \neq \emptyset .
$$

If $z \in \widehat{\mathscr{K}}$, one has

$$
\begin{aligned}
F(z) & =F(z)-\frac{1}{2}\left\langle F^{\prime}(z), z\right\rangle \\
& =\int_{\mathbb{R}^{N}} \frac{1}{2} h_{\infty}(|z|)|z|^{2}-H_{\infty}(z) d x \\
& =\int_{\mathbb{R}^{N}} \widetilde{H}_{\infty}(z) d x \geq 0,
\end{aligned}
$$

which implies $\widehat{C} \geq 0$. If $\widehat{C}=0$, let $z_{n} \in \widehat{\mathscr{K}} \backslash\{0\}$ be such that $F\left(z_{n}\right) \rightarrow 0$ as $n \rightarrow \infty$. Then $\left\{z_{n}\right\}$ is $(C)_{0}$-sequence. By Lemma 12, we can assume that $z_{n} \rightarrow z \in E$. Then

$$
\begin{aligned}
F\left(z_{n}\right) & =F\left(z_{n}\right)-\frac{1}{2}\left\langle F^{\prime}\left(z_{n}\right), z_{n}\right\rangle \\
& =\int_{\mathbb{R}^{N}} \frac{1}{2} h_{\infty}\left(\left|z_{n}\right|\right)\left|z_{n}\right|^{2}-H_{\infty}\left(z_{n}\right) d x \\
& =\int_{\mathbb{R}^{N}} \widetilde{H}_{\infty}\left(z_{n}\right) d x \longrightarrow 0 .
\end{aligned}
$$

By Lemma 8(i), we see that, for any $\varepsilon>0$, there is $1>r=$ $r(\varepsilon)>0$ such that

$$
h_{\infty}(|z|) \leq \varepsilon, \quad \text { whenever }|z|<r .
$$

It follows from Lemma 8(iv) that there exists constant $R>$ $r>0$ such that

$$
h_{\infty}(|z|) \leq c_{2}^{1 / \tau}\left|\widetilde{H}_{\infty}(z)\right|^{1 / \tau}, \quad \text { whenever }|z|>R
$$

By the continuity of $\widetilde{H}_{\infty}(z)$, there exists $M_{0}=M_{0}(r, R)>0$ such that

$$
\widetilde{H}_{\infty}(z) \geq M_{0}, \quad \text { whenever } r \leq|z| \leq R .
$$

Note that $h_{\infty}(|z|) \leq c_{1}\left(1+|z|^{p-2}\right)$ for some $p \in\left(2,2^{*}\right)$. Hence, there exists $M_{1}=M_{1}(R)$ such that for all $r \leq|z| \leq R$

$$
\begin{aligned}
h_{\infty}(|z|) & \leq M_{1} \leq \frac{1}{\left|\widetilde{H}_{\infty}(z)\right|^{1 / \tau}} M_{1}\left|\widetilde{H}_{\infty}(z)\right|^{1 / \tau} \\
& \leq M_{1} M_{0}^{-1 / \tau}\left|\widetilde{H}_{\infty}(z)\right|^{1 / \tau} .
\end{aligned}
$$

Denote $C_{\varepsilon}=c_{2}^{1 / \tau}+M_{1} M_{0}^{-1 / \tau}$; we obtain

$$
h_{\infty}(|z|) \leq \varepsilon+C_{\varepsilon}\left|\widetilde{H}_{\infty}(z)\right|^{1 / \tau}, \quad \forall z \in R^{2} .
$$

By Hölder inequality $\left(1 / \tau+1 / \tau^{\prime}=1\right)$, we have

$$
\begin{aligned}
\left\|z_{n}\right\|^{2}=\int_{\mathbb{R}^{N}} h_{\infty}\left(\left|z_{n}\right|\right) z_{n}\left(z_{n}^{+}-z_{n}^{-}\right) d x \\
\leq \varepsilon\left|z_{n}\right|_{2}^{2}+C_{\varepsilon} \int_{\mathbb{R}^{N}}\left|z_{n}^{+}-z_{n}^{-}\right|\left|z_{n}\right|\left|\widetilde{H}_{\infty}\left(z_{n}\right)\right|^{1 / \tau} d x \\
\leq \varepsilon\left|z_{n}\right|_{2}^{2} \\
\quad+C_{\varepsilon}\left(\int_{\mathbb{R}^{N}} \widetilde{H}_{\infty}\left(z_{n}\right) d x\right)^{1 / \tau}\left(\int_{\mathbb{R}^{N}}\left|z_{n}\right|^{2 \tau^{\prime}} d x\right)^{1 / \tau^{\prime}} \\
\leq \varepsilon a_{2}^{2}\left\|z_{n}\right\|^{2}+a_{2 \tau^{\prime}}^{2} C_{\varepsilon}\left(\int_{\mathbb{R}^{N}} \widetilde{H}_{\infty}\left(z_{n}\right) d x\right)^{1 / \tau}\left\|z_{n}\right\|^{2} .
\end{aligned}
$$

Hence $1 \leq \varepsilon a_{2}^{2}+o(1)$, a contradiction. Hence $\widehat{C}>0$. Finally, we show that there is $z \in \widehat{\mathscr{K}}$ with $F(z)=\widehat{C}>0$. Let 
$z_{n} \in \widehat{\mathscr{K}} \backslash\{0\}$ be such that $F\left(z_{n}\right) \rightarrow \widehat{C}$. Then, as before, $\left\{z_{n}\right\}$ is bounded, and one may assume $z_{n} \rightarrow z \in \widehat{\mathscr{K}} \backslash\{0\}$. Now

$$
\begin{aligned}
\widehat{C} & =\lim _{n \rightarrow \infty} F\left(z_{n}\right)=\lim _{n \rightarrow \infty} \int_{\mathbb{R}^{N}} \widetilde{H}_{\infty}\left(z_{n}\right) d x \\
& \geq \int_{\mathbb{R}^{N}} \widetilde{H}_{\infty}(z) d x=F(z) \geq \widehat{C} ;
\end{aligned}
$$

that is, $F(z)=\widehat{C}$. This completes the proof.

Definition 14 (see Ackermann [28]). A mapping $f$ from Banach space $X$ to another Banach space $Y$ is called BLsplit, if for every weakly convergent sequence $\left\{x_{n}\right\} \subset X$ with $x_{n} \rightarrow x$ it holds that $f\left(x_{n}\right)-f\left(x_{n}-x\right) \rightarrow f(x)$ in $Y$.

In what follows, we use the idea of $[21,28]$. For fixed $w \in$ $E^{+}$, we introduce the functional $\phi_{w}: E^{-} \rightarrow \mathbb{R}$ by

$$
\begin{aligned}
\phi_{w}(y) & :=F(w+y) \\
& =\frac{1}{2}\left(\|w\|^{2}-\|y\|^{2}\right)-\Psi_{\infty}(w+y) .
\end{aligned}
$$

Hence one has

$$
\begin{aligned}
\phi_{w}^{\prime \prime}(y)\left[z^{-}, z^{-}\right]=-\left\|z^{-}\right\|^{2}-\Psi_{\infty}^{\prime \prime}(w+y)\left[z^{-}, z^{-}\right] \\
=-\left\|z^{-}\right\|^{2}-\int_{\mathbb{R}^{N}} \frac{h_{\infty}^{\prime}(|w+y|)}{|w+y|}\left((w+y) z^{-}\right)^{2} d x \\
-\int_{\mathbb{R}^{N}} h_{\infty}(|w+y|)\left|z^{-}\right|^{2} d x,
\end{aligned}
$$

for all $y, z^{-} \in E^{-}$, which implies that $\phi_{w}(\cdot)$ is strictly concave. Moreover

$$
\phi_{w}(y) \leq \frac{1}{2}\left(\|w\|^{2}-\|y\|^{2}\right),
$$

which implies that $\phi_{w}(y) \rightarrow-\infty$ as $\|y\| \rightarrow \infty$. Now, it follows from Lemma 9 that $\phi_{w}$ is weakly sequentially upper semicontinuous. Hence, there is a unique strict maximum point $\xi(w)$ for $\phi_{w}(\cdot)$, which is also the only critical point of $\phi_{w}$ on $E^{-}$and satisfies

$$
\begin{aligned}
& y \neq \xi(w) \Longleftrightarrow \\
& F(w+y)<F(w+\xi(w)), \\
& \left\langle\phi_{w}^{\prime}(\xi(w)), z^{-}\right\rangle=0, \\
& \text { i.e., }\left(\xi(w), z^{-}\right)+\left\langle\Psi_{\infty}^{\prime}(w+\xi(w)), z^{-}\right\rangle=0,
\end{aligned}
$$

for all $w \in E^{+}, y, z^{-} \in E^{-}$. Now, we define the reduced functional $\mathscr{R}: E^{+} \rightarrow \mathbb{R}$ by

$$
\begin{aligned}
\mathscr{R}(w) & :=F(w+\xi(w)) \\
& =\frac{1}{2}\|w\|^{2}-\frac{1}{2}\|\xi w\|^{2}-\Psi_{\infty}(w+\xi(w)) .
\end{aligned}
$$

We have the following lemma.
Lemma 15. $w \in E^{+}$is a critical point of $\mathscr{R}$ if and only if $w+$ $\xi(w)$ is a critical point of F. Moreover, the following conclusions hold:

(1) $\xi \in C^{1}\left(E^{+}, E^{-}\right)$and $\xi(0)=0$.

(2) $\xi$ is a bounded map.

(3) $\xi,\|\xi(w)\|^{2},|\xi(w)|_{2}^{2}$ are all BL-splits.

(4) $\xi\left(w_{n}\right) \rightarrow \xi(w)$ in $E^{-}$if $w_{n}-w$ in $E^{+}$.

(5) $\xi$ is $\mathbb{Z}^{N}$-invariant, i.e., $\xi(a * w)=\xi(w)$ for all $a \in \mathbb{Z}^{N}$.

Proof. It follows from $\left(H_{7}\right)$ that $H_{\infty} \in C^{2}\left(\mathbb{R}^{N} \times \mathbb{R}^{2},[0, \infty)\right)$ and

$$
\begin{aligned}
H_{\infty}^{\prime \prime}(z) z_{1} \cdot z_{1} & =\left(h_{\infty}^{\prime}(|z|)|z| z_{1}+h_{\infty}(|z|) z_{1}\right) \cdot z_{1} \\
& \geq 0, \quad \forall z, z_{1} \in \mathbb{R}^{2} .
\end{aligned}
$$

Hence, combining with $\left(V_{1}\right),\left(B_{0}\right)$, and $\left(B_{1}\right)$, we know that all of the conditions of Lemma 2.6 in [19] hold. So, by Lemma 2.6 in [19], the desired conclusions can be obtained.

By Lemma 15 , the critical points of $\mathscr{R}$ and $F$ are in oneto-one correspondence via the injective map $w \rightarrow w+\xi(w)$ from $E^{+}$into $E$. Consequently, let

$$
\widehat{\mathscr{K}}^{+}:=\left\{w \in E^{+}: \mathscr{R}^{\prime}(w)=0\right\} .
$$

Then

$$
\widehat{\mathscr{K}}=\left\{w+\xi(w): w \in \widehat{\mathscr{K}}^{+}\right\}
$$

In particular

$$
\widehat{C}=\inf \left\{\mathscr{R}(w): w \in \widehat{\mathscr{K}}^{+}\right\} .
$$

Lemma 16. The sequence $\left\{w_{n}\right\} \subset E^{+}$is a bounded $(C)_{c^{-}}$ sequence of $\mathscr{R}$ if and only if $\left\{w_{n}+\xi\left(w_{n}\right)\right\} \subset E$ is a bounded $(C)_{c}$-sequence of $F$.

Proof. Let $\left\{w_{n}\right\} \subset E^{+}$be a $(C)_{c}$ sequence of $\mathscr{R}$; that is, $\mathscr{R}\left(w_{n}\right) \rightarrow c$ and $\left(1+\left\|w_{n}\right\|\right) \mathscr{R}^{\prime}\left(w_{n}\right) \rightarrow 0$. Since $H_{\infty} \geq 0$, we have

$$
\begin{aligned}
\left\|\xi\left(w_{n}\right)\right\|^{2}= & \left\|w_{n}\right\|^{2}-2 \int_{R^{N}} H_{\infty}\left(w_{n}+\xi\left(w_{n}\right)\right) d x-2 c \\
& +o(1) \leq\left(\left\|w_{n}\right\|+C\right)^{2} .
\end{aligned}
$$


By (81) and Lemma 15, for all $z=z^{+}+z^{-} \in E$, we have $\xi^{\prime}\left(w_{n}\right) z^{+} \in E^{-}$and

$$
\begin{aligned}
\left\langle\mathscr{R}^{\prime}\right. & \left.\left(w_{n}\right), z^{+}\right\rangle \\
= & \left(w_{n}, z^{+}\right)-\left(\xi\left(w_{n}\right), \xi^{\prime}\left(w_{n}\right) z^{+}\right) \\
& -\int_{\mathbb{R}^{N}} H_{\infty}^{\prime}\left(w_{n}+\xi\left(w_{n}\right)\right)\left(z^{+}+\xi^{\prime}\left(w_{n}\right) z^{+}\right) d x \\
= & \left(w_{n}, z^{+}\right)-\int_{\mathbb{R}^{N}} H_{\infty}^{\prime}\left(w_{n}+\xi\left(w_{n}\right)\right) z^{+} d x \\
= & \left(w_{n}, z^{+}\right)-\left(\xi\left(w_{n}\right), z^{-}\right) \\
& -\int_{\mathbb{R}^{N}} H_{\infty}^{\prime}\left(w_{n}+\xi\left(w_{n}\right)\right)\left(z^{+}+z^{-}\right) d x \\
= & \left\langle F^{\prime}\left(w_{n}+\xi\left(w_{n}\right)\right), z\right\rangle .
\end{aligned}
$$

Hence, one has

$$
\begin{aligned}
\left\|\mathscr{R}^{\prime}\left(w_{n}\right)\right\| & =\sup _{\left\|z^{+}\right\| \leq 1}\left\langle\mathscr{R}^{\prime}\left(w_{n}\right), z^{+}\right\rangle \\
& =\sup _{\left\|z^{+}\right\| \leq 1}\left\langle F^{\prime}\left(w_{n}+\xi\left(w_{n}\right)\right), z^{+}\right\rangle \\
& \leq \sup _{\left\|z^{+}\right\| \leq 1}\left\|F^{\prime}\left(w_{n}+\xi\left(w_{n}\right)\right)\right\| \cdot\left\|z^{+}\right\| \\
& \leq\left\|F^{\prime}\left(w_{n}+\xi\left(w_{n}\right)\right)\right\| .
\end{aligned}
$$

Similarly

$$
\begin{aligned}
\left\|F^{\prime}\left(w_{n}+\xi\left(w_{n}\right)\right)\right\| & =\sup _{\|z\| \leq 1}\left\langle F^{\prime}\left(w_{n}+\xi\left(w_{n}\right)\right), z\right\rangle \\
& \leq \sup _{\left\|z^{+}\right\| \leq 1}\left\langle\mathscr{R}^{\prime}\left(w_{n}\right), z^{+}\right\rangle \\
& \leq\left\|\mathscr{R}^{\prime}\left(w_{n}\right)\right\| .
\end{aligned}
$$

Consequently

$$
\left\|\mathscr{R}^{\prime}\left(w_{n}\right)\right\|=\left\|F^{\prime}\left(w_{n}+\xi\left(w_{n}\right)\right)\right\| .
$$

Hence

$$
\begin{aligned}
& \left\|\xi\left(w_{n}\right)\right\|\left\|F^{\prime}\left(w_{n}+\xi\left(w_{n}\right)\right)\right\| \\
& \quad \leq\left(\left\|w_{n}\right\|+C\right)\left\|F^{\prime}\left(w_{n}+\xi\left(w_{n}\right)\right)\right\| \\
& \quad=\left(\left\|w_{n}\right\|+C\right)\left\|\mathscr{R}^{\prime}\left(w_{n}\right)\right\| \longrightarrow 0, \\
& \left(1+\left\|w_{n}+\xi\left(w_{n}\right)\right\|\right)\left\|F^{\prime}\left(w_{n}+\xi\left(w_{n}\right)\right)\right\| \\
& \quad \leq\left(1+\left\|w_{n}\right\|+\left\|\xi\left(w_{n}\right)\right\|\right)\left\|F^{\prime}\left(w_{n}+\xi\left(w_{n}\right)\right)\right\| \longrightarrow 0 .
\end{aligned}
$$

That is, $\left\{w_{n}+\xi\left(w_{n}\right)\right\}$ is $(C)_{c}$ sequence of $F$. The inverse is obvious. By Lemma 15, the boundedness of $\xi$ yields the equivalence between the boundedness of $\left\{w_{n}\right\}$ and $\left\{w_{n}+\right.$ $\left.\xi\left(w_{n}\right)\right\}$. This completes the proof.
Next, we discuss the mountain pass geometry of the reduce functional $\mathscr{R}$. One has the following Lemma.

Lemma 17. $\mathscr{R}$ possesses the mountain pass geometry:

(1) There is $\rho>0$ such that inf $\mathscr{R}\left(E^{+} \cap \partial B_{\rho}\right)>0$, where $\partial B_{\rho}=\{z \in E:\|z\|=\rho\}$.

(2) There is some e $\in E^{+} \backslash \bar{B}_{\rho}(0)$ such that $\mathscr{R}(e) \leq 0$.

Proof. (1) By (31) and (80), for any $w \in E^{+}$, we have

$$
\begin{aligned}
\mathscr{R}(w) & =\frac{1}{2}\left(\|w\|^{2}-\|\xi(w)\|^{2}\right)-\Psi_{\infty}(w+\xi(w)) \\
& =\frac{1}{2}\|w\|^{2}+(F(w+\xi(w))-F(w))-\Psi_{\infty}(w) \\
& \geq \frac{1}{2}\|w\|^{2}-\Psi_{\infty}(w) \\
& \geq \frac{1}{2}\|w\|^{2}-\frac{\varepsilon}{2}|w|_{2}^{2}-\frac{C(\varepsilon)}{p}|w|_{p}^{p} \\
& \geq\left(\frac{1}{2}-C \varepsilon\right)\|w\|^{2}-C\|w\|^{p} .
\end{aligned}
$$

Hence, for small $\rho>0$, conclusion (1) holds.

(2) Similar to the proof of Lemma 10, we can obtain that

$$
\begin{aligned}
& \mathscr{R}(w) \longrightarrow-\infty, \\
& \text { as }\|w\| \longrightarrow \infty,
\end{aligned}
$$

$$
w \in E_{W}
$$

Hence conclusion (2) holds.

Remark 18. Lemma 17 implies that 0 is an isolated critical point of $\mathscr{R}$. Therefore there is a $v>0$ such that $\|w\| \geq v$ for all $w \in K_{\mathscr{R}}$, where $K_{\mathscr{R}}:=\left\{w \in E^{+} \mid w \neq 0\right.$ and $\left.\mathscr{R}^{\prime}(w)=0\right\}$.

Remark 19. If we set

$$
\mathscr{N}^{+}:=\left\{w \in E^{+} \backslash\{0\}:\left\langle\mathscr{R}^{\prime}(w), w\right\rangle=0\right\},
$$

then, by Lemma 4.7 in [18], we have that,. for each $w \in E^{+} \backslash$ $\{0\}$, there is a unique $s(w)>0$ such that $s(w) w \in \mathscr{N}^{+}$and $\mathscr{R}(s(w) w)=\sup _{s \geq 0} \mathscr{R}(s w)$.

Lemma 20. Let $w \in \widehat{\mathscr{K}}^{+}$be such that $\mathscr{R}(w)=\widehat{C}$ and set $E_{w}=E^{-} \oplus \mathbb{R}^{+} w$. Then

$$
\sup _{z \in E_{w}} F(z) \leq \mathscr{R}(w)=\widehat{C} .
$$

Proof. For any $z=z^{-}+s w \in E_{w}$, by (80), we obtain

$$
F(z) \leq F(s w+\xi(s w))=\mathscr{R}(s w) .
$$

Since $w \in \widehat{\mathscr{K}}^{+}$, we have $w \in \mathscr{N}^{+}$. Hence, combining with Remark 19, we obtain $\mathscr{R}(w)=\sup _{s \geq 0} \mathscr{R}(s w)$. Consequently, one has

$$
\sup _{z \in E_{w}} F(z) \leq \sup _{s \geq 0} \mathscr{R}(s w)=\mathscr{R}(w)=\widehat{C} .
$$




\section{Proof of the Main Result}

In this section we give the proof of Theorem 1 . Let $\mathscr{K}:=\{z \in$ $\left.E: \Phi^{\prime}(z)=0\right\}$ be the set of critical points for $\Phi$. First, we study the linking structure for the functional $\Phi$. Similar to the proof of Lemma 9, we have the following three lemmas.

Lemma 21. $\Psi$ is nonnegative and weakly sequentially lower semicontinuous.

Lemma 22. $\Phi^{\prime}$ is weakly sequentially continuous.

Lemma 23. There exists $r>0$ such that $\kappa_{1}:=\inf \Phi\left(S_{r} \cap E^{+}\right)>$ 0 , where $S_{r}:=\{z \in E:\|z\|=r\}$.

Lemma 24. There is $\rho>r>0$ such that for any $e \in E^{+}$with $\|e\|=1$ and $E_{e}=E^{-} \oplus \mathbb{R}^{+} e$

$$
\Phi(z)<0, \quad \forall z \in E_{e} \backslash B_{\rho}
$$

Proof. For any $z \in E_{e}$, by $\left(H_{7}\right)$, we have

$$
\begin{aligned}
\Phi(z) & =\frac{1}{2}\left(\left\|z^{+}\right\|^{2}-\left\|z^{-}\right\|^{2}\right)-\int_{\mathbb{R}^{N}} H(x, z) d x \\
& \leq \frac{1}{2}\left(\left\|z^{+}\right\|^{2}-\left\|z^{-}\right\|^{2}\right)-\int_{\mathbb{R}^{N}} H_{\infty}(z) d x \\
& =F(z) .
\end{aligned}
$$

Thus, the conclusion follows easily from Lemma 10.

In particular, set $z_{0}^{+} \in \widehat{\mathscr{K}}^{+}$with $\mathscr{R}\left(z_{0}^{+}\right)=\widehat{C}, e=z_{0}^{+}$, and $Q=\left\{z=z^{-}+s z_{0}^{+}: z^{-} \in E^{-}, s \geq 0,\|z\| \leq \rho\right\}$, where $\rho$ is given in Lemma 24. Then we have the following lemma.

Lemma 25. $d=\sup \left\{\Phi(z): z \in E_{e}\right\}=\sup \Phi(Q)<\widehat{C}$.

Proof. By Lemmas 23 and 24, we have $d=\sup \Phi(Q) \geq \kappa_{1}$. Since $\Phi$ is weakly upper semicontinuous on $E_{e}$, there is some $z_{1} \in Q$ with $z_{1} \neq 0$ such that $\sup \Phi(Q)=\Phi\left(z_{1}\right)$. From $\left(H_{7}\right)$ and Lemma 20, we obtain

$$
\begin{aligned}
d & =\sup \left\{\Phi(z): z \in E_{e}\right\}=\sup \Phi(Q)=\Phi\left(z_{1}\right) \\
& <F\left(z_{1}\right) \leq \sup F(Q) \leq \mathscr{R}\left(z_{0}^{+}\right)=\widehat{C} .
\end{aligned}
$$

In what follows, we discuss $(C)_{c}$ sequences of $\Phi$. Firstly we have the following.

Lemma 26. Any $(C)_{c}$-sequence for $\Phi$ is bounded.

Proof. The proof is similar to Lemma 12. We omit the details here.

Lemma 27 (see [23, Theorem A.2]). Let $\Omega$ be an open set in $\mathbb{R}^{N}$ and $f \in C(\Omega \times \mathbb{R}, \mathbb{R})$ be a function such that $|f(x, u)| \leq$ $a\left(|u|^{r}+|u|^{s}\right)$ for some $a>0$ and $1 \leq r<s<\infty$. Suppose $s \leq p<\infty, r \leq q<\infty, q>1,\left\{u_{n}\right\}$ is a bounded sequence in $L^{p}(\Omega) \cap L^{q}(\Omega)$, and $u_{n} \rightarrow u$ a.e. in $\Omega$ and in $L^{p}\left(\Omega \cap B_{\rho}\right) \cap L^{q}\left(\Omega \cap B_{\rho}\right)$ for all $\rho>0$. Then, passing to a subsequence, there exists a sequence $\left\{v_{n}\right\}$ such that $v_{n} \rightarrow u$ in $L^{p}(\Omega) \cap L^{q}(\Omega)$ and

$$
\begin{aligned}
f\left(x, u_{n}\right)-f\left(x, u_{n}-v_{n}\right)-f(x, u) & \longrightarrow 0 \\
& \quad \text { in } L^{q / r}(\Omega)+L^{p / s}(\Omega),
\end{aligned}
$$

where $v_{n}(x)=\chi\left(2|x| / r_{n}\right) u(x)$ and $\chi \in C^{\infty}(\mathbb{R},[0,1])$ is such that $\chi(t)=1$ for $t \leq 1, \chi(t)=0$ for $t \geq 2, r_{n}>0$ is a sequence of constants with $r_{n} \rightarrow \infty$ as $n \rightarrow \infty$, the space $L^{p}(\Omega) \cap L^{q}(\Omega)$ with the norm

$$
\|u\|_{p \wedge q}:=\|u\|_{p}+\|u\|_{q}
$$

and the space $L^{p}(\Omega)+L^{q}(\Omega)$ with the norm

$$
\begin{aligned}
& \|u\|_{p \vee q}:=\inf \left\{\|v\|_{p}+\|w\|_{q}: v \in L^{p}(\Omega), w\right. \\
& \left.\in L^{q}(\Omega), u=v+w\right\} .
\end{aligned}
$$

Lemma 28. Let $\left\{z_{n}\right\} \subset E$ be $(C)_{c}$-sequence of $\Phi$. Then, passing to a subsequence if necessary, we can assume that $z_{n} \rightarrow z_{0}$ in E. Furthermore, either

(i) $z_{n} \rightarrow z_{0} \in$ E or

(ii) $c \geq \widehat{C}$ and there exist a positive integer $l \leq[c / \widehat{C}]$, $y_{1}, \ldots, y_{l} \in \widehat{\mathscr{K}} \backslash\{0\}$ and sequences $\left\{g_{n}^{i}\right\} \subset Z^{N}, i=$ $1,2, \ldots, l$, such that, after extraction of a subsequence of $\left\{z_{n}\right\}$

$$
\begin{gathered}
\left\|z_{n}-\widetilde{z}_{n}-\sum_{i=1}^{l} g_{n}^{i} * y_{i}\right\| \longrightarrow 0, \\
\Phi\left(z_{0}\right)+\sum_{i=1}^{l} F\left(y_{i}\right)=c
\end{gathered}
$$

where $\widetilde{z}_{n}(x)=\eta(2|x| / n) z_{0}(x)$ and $\eta \in C^{\infty}(\mathbb{R},[0,1])$ is a cutoff function such that $\eta(t)=1$ for $t \leq 1, \eta(t)=0$ for $t \geq 2$.

Proof. First, Lemma 26 implies that any $(C)_{c}$ sequence of $\Phi$ is bounded; hence it is a bounded (PS) ${ }_{c}$ sequence. Passing to a subsequence if necessary, we can assume that $z_{n} \rightarrow z_{0} \in E$ with $\Phi^{\prime}\left(z_{0}\right)=0, z_{n}(x) \rightarrow z_{0}(x)$ a.e. in $\mathbb{R}^{N}$ and $z_{n} \rightarrow z_{0}$ in $L_{\text {loc }}\left(\mathbb{R}^{N}, \mathbb{R}^{2}\right)$. Let $\eta \in C^{\infty}(\mathbb{R},[0,1])$ be a cut-off function such that $\eta(t)=1$ for $t \leq 1, \eta(t)=0$ for $t \geq 2$ and define $\widetilde{z}_{n}(x)=$ $\eta(2|x| / n) z_{0}(x)$; then $\widetilde{z}_{n} \rightarrow z_{0}$ in $E$. Indeed, by Lemma $5, z_{0} \in$ $E$ implies that for any $\varepsilon>0$ there is a corresponding $\rho=$ $\rho(\varepsilon)>0$ such that

$$
\begin{aligned}
& \int_{\mathbb{R}^{N} \backslash B_{\rho}(0)}\left|z_{0}\right|^{2} d x \leq \varepsilon, \\
& \int_{\mathbb{R}^{N} \backslash B_{\rho}(0)}\left|\nabla z_{0}\right|^{2} d x \leq \varepsilon,
\end{aligned}
$$




$$
\begin{aligned}
\| \tilde{z}_{n}- & z_{0}\left\|^{2} \leq C\right\| \tilde{z}_{n}-z_{0} \|_{H^{1}\left(\mathbb{R}^{N}, \mathbb{R}^{2}\right)}^{2} \\
\leq & C \int_{\mathbb{R}^{N}}\left|\nabla\left(\widetilde{z}_{n}-z_{0}\right)\right|^{2} d x+C \int_{\mathbb{R}^{N}}\left|\tilde{z}_{n}-z_{0}\right|^{2} d x \\
= & C \int_{\mathbb{R}^{N}}\left|\nabla\left(\eta\left(\frac{2|x|}{n}\right) z_{0}-z_{0}\right)\right|^{2} d x \\
& +C \int_{\mathbb{R}^{N}}\left|\eta\left(\frac{2|x|}{n}\right) z_{0}-z_{0}\right|^{2} d x \\
\leq & C \int_{\mathbb{R}^{N}}\left(\eta\left(\frac{2|x|}{n}\right)-1\right)^{2}\left|\nabla z_{0}\right|^{2} d x \\
& +\frac{4 C}{n^{2}} \int_{\mathbb{R}^{N}}\left(\eta^{\prime}\left(\frac{2|x|}{n}\right)\right)^{2}\left|z_{0}\right|^{2} d x \\
& +C \int_{\mathbb{R}^{N}}\left(\eta\left(\frac{2|x|}{n}\right)-1\right)^{2}\left|z_{0}\right|^{2} d x \\
\leq & C \int_{B_{\rho}(0)}\left(\eta\left(\frac{2|x|}{n}\right)-1\right)^{2}\left|\nabla z_{0}\right|^{2} d x \\
& +\frac{4 C}{n^{2}} \int_{\mathbb{R}^{N}}\left(\eta^{\prime}\left(\frac{2|x|}{n}\right)\right)^{2}\left|z_{0}\right|^{2} d x \\
& +C \int_{B_{\rho}(0)}\left(\eta\left(\frac{2|x|}{n}\right)-1\right)^{2}\left|z_{0}\right|^{2} d x+C \varepsilon .
\end{aligned}
$$

Hence, using the Lebesgue dominated convergence theorem, we obtain

$$
\left\|\widetilde{z}_{n}-z_{0}\right\| \longrightarrow 0, \text { as } n \longrightarrow \infty .
$$

Now we set $z_{n}^{1}=z_{n}-\widetilde{z}_{n}$. Then, by $z_{n} \rightarrow z_{0}$, one has $z_{n}^{1} \rightarrow 0$. Moreover, we claim that $z_{n}^{1}$ is a bounded (PS) $)_{\mathcal{c}_{1}}$-sequence for $F$ with $c_{1}=c-\Phi\left(z_{0}\right)$. In fact, by $\left(H_{7}\right)$, for any $\varepsilon>0$, there is a $\rho_{1}>0$ such that

$$
\left|h(x, s)-h_{\infty}(s)\right| \leq \varepsilon, \quad \text { whenever }|x|>\rho_{1},
$$

uniformly in bounded set of $s$. On the one hand, by (19), taking $r=2, s=p$, and $q=2$ in Lemma 27, we know that

$$
\begin{aligned}
\int_{\mathbb{R}^{N}} H\left(x, z_{n}\right) d x= & \int_{\mathbb{R}^{N}} H\left(x, z_{n}^{1}\right) d x \\
& +\int_{\mathbb{R}^{N}} H\left(x, z_{0}\right) d x+o(1) .
\end{aligned}
$$

On the other hand, by (18), taking $r=1, s=p-1$ and $q=2$. It follows from Lemma 27 that

$$
g_{n} \longrightarrow 0, \quad \text { in } L^{2}\left(\mathbb{R}^{N}, \mathbb{R}^{2}\right)+L^{p /(p-1)}\left(\mathbb{R}^{N}, \mathbb{R}^{2}\right),
$$

where

$$
\begin{aligned}
g_{n}(x):= & H_{z}\left(x, z_{n}(x)\right)-H_{z}\left(x, z_{n}^{1}(x)\right) \\
& -H_{z}\left(x, z_{0}(x)\right) .
\end{aligned}
$$

Hence, for each $\varphi \in E$ with $\|\varphi\|=1$, one has

$$
\begin{aligned}
& \left|\int_{\mathbb{R}^{N}}\left[H_{z}\left(x, z_{n}\right)-H_{z}\left(x, z_{n}^{1}\right)-H_{z}\left(x, z_{0}\right)\right] \varphi d x\right| \\
& \quad \leq\left\|g_{n}\right\|_{2 \vee p^{\prime}}\|\varphi\|_{2 \wedge p} \leq C\left\|g_{n}\right\|_{2 \vee p^{\prime}},
\end{aligned}
$$

where $p^{\prime}=p /(p-1)$ and the constant $C>0$ is independent of $\varphi$. Consequently

$$
\begin{aligned}
& \sup _{\|\varphi\|=1} \int_{\mathbb{R}^{N}}\left[H_{z}\left(x, z_{n}\right)-H_{z}\left(x, z_{n}^{1}\right)-H_{z}\left(x, z_{0}\right)\right] \varphi d x \\
& \quad=o(1) .
\end{aligned}
$$

For any $z \in E$, observe that

$$
\begin{aligned}
\Phi(z) & =\frac{1}{2}(A z, z)_{2}-\int_{R^{N}} H(x, z) d x, \\
A z_{n} & =A z_{n}^{1}+A z_{0}+o(1) .
\end{aligned}
$$

Hence, it follows from (110) and (114) that

$$
\begin{gathered}
\Phi\left(z_{n}\right)=\Phi\left(z_{n}^{1}\right)+\Phi\left(z_{0}\right)+o(1), \\
\Phi^{\prime}\left(z_{n}\right)=\Phi^{\prime}\left(z_{n}^{1}\right)+\Phi^{\prime}\left(z_{0}\right)+o(1),
\end{gathered}
$$

and then

$$
\begin{aligned}
\Phi\left(z_{n}^{1}\right) & \longrightarrow c-\Phi\left(z_{0}\right), \\
\Phi^{\prime}\left(z_{n}^{1}\right) & \longrightarrow 0,
\end{aligned}
$$

as $n \rightarrow \infty$. Consequently, by (19), (31), and (117), for large $n$, we obtain

$$
\begin{aligned}
& c-\Phi\left(z_{0}\right)+o(1)=\Phi\left(z_{n}^{1}\right) \leq F\left(z_{n}^{1}\right)=\Phi\left(z_{n}^{1}\right) \\
& +\int_{\mathbb{R}^{N}} H\left(x, z_{n}^{1}\right) d x-\int_{\mathbb{R}^{N}} H_{\infty}\left(z_{n}^{1}\right) d x \leq c \\
& - \\
& -\Phi\left(z_{0}\right)+o(1)+\int_{|x| \leq \rho_{1}} \mid H\left(x, z_{n}^{1}\right) \\
& -H_{\infty}\left(z_{n}^{1}\right)\left|d x+\int_{|x|>\rho_{1}}\right| H\left(x, z_{n}^{1}\right) \\
& \quad-H_{\infty}\left(z_{n}^{1}\right) \mid d x \leq c-\Phi\left(z_{0}\right)+o(1) \\
& +\int_{|x| \leq \rho_{1}}\left|H\left(x, z_{n}^{1}\right)-H_{\infty}\left(z_{n}^{1}\right)\right| d x \\
& +\int_{|x|>\rho_{1}} \int_{0}^{1}\left|h\left(x, \theta\left|z_{n}^{1}\right|\right)-h_{\infty}\left(\theta\left|z_{n}^{1}\right|\right)\right| \\
& \quad \cdot\left|z_{n}^{1}\right|^{2} d \theta d x \leq c-\Phi\left(z_{0}\right)+o(1) \\
& +\int_{|x| \leq \rho_{1}}\left|H\left(x, z_{n}^{1}\right)-H_{\infty}\left(z_{n}^{1}\right)\right| d x \\
& \quad+\varepsilon \int_{|x|>\rho_{1}}\left|z_{n}^{1}\right|^{2} d x \leq c-\Phi\left(z_{0}\right)+o(1) \\
& +C \int_{|x| \leq \rho_{1}}\left(\left|z_{n}^{1}\right|^{2}+\left|z_{n}^{1}\right|^{p}\right) d x+\varepsilon \int_{|x|>\rho_{1}}\left|z_{n}^{1}\right|^{2} d x .
\end{aligned}
$$


Consequently

$$
F\left(z_{n}^{1}\right) \longrightarrow c-\Phi\left(z_{0}\right)
$$

In what follows, we claim that

$$
\Psi^{\prime}\left(z_{n}^{1}\right)-\Psi_{\infty}^{\prime}\left(z_{n}^{1}\right) \longrightarrow 0, \quad \text { as } n \longrightarrow \infty .
$$

Indeed, since $z_{n}^{1} \rightarrow 0$ in $L_{\text {loc }}^{p}\left(R^{N}\right)$ for $2 \leq p<2^{*}$, by (18), (30), and Theorem A.2 in [27], we have

$$
\begin{gathered}
H_{z}\left(x, z_{n}^{1}\right) \longrightarrow 0, \quad \text { in } L_{\text {loc }}^{p / p-1}\left(\mathbb{R}^{N}, \mathbb{R}^{2}\right), \\
H_{\infty}^{\prime}\left(z_{n}^{1}\right) \longrightarrow 0, \quad \text { in } L_{\text {loc }}^{p / p-1}\left(\mathbb{R}^{N}, \mathbb{R}^{2}\right) .
\end{gathered}
$$

Hence, for any $w \in E$ with $\|w\|=1$, it follows from (109) and (121) that

$$
\begin{aligned}
& \left|\left\langle\Psi^{\prime}\left(z_{n}^{1}\right)-\Psi_{\infty}^{\prime}\left(z_{n}^{1}\right), w\right\rangle\right| \\
& =\left|\int_{\mathbb{R}^{N}}\left[H_{z}\left(x, z_{n}^{1}\right)-H_{\infty}^{\prime}\left(x, z_{n}^{1}\right)\right] w d x\right| \\
& =\int_{|x| \leq \rho_{1}}\left|H_{z}\left(x, z_{n}^{1}\right)-H_{\infty}^{\prime}\left(z_{n}^{1}\right)\right||w| d x \\
& +\int_{|x|>\rho_{1}}\left|H_{z}\left(x, z_{n}^{1}\right)-H_{\infty}^{\prime}\left(z_{n}^{1}\right)\right||w| d x \\
& \leq\left[\left(\int_{|x| \leq \rho_{1}}\left|H_{z}\left(x, z_{n}^{1}\right)\right|^{p /(p-1)} d x\right)^{(p-1) / p}\right. \\
& \left.+\left(\int_{|x| \leq \rho_{1}}\left|H_{\infty}^{\prime}\left(z_{n}^{1}\right)\right|^{p /(p-1)} d x\right)^{(p-1) / p}\right] \\
& \cdot\left(\int_{|x| \leq \rho_{1}}|w|^{p} d x\right)^{1 / p}+\int_{|x|>\rho_{1}}\left|h_{z}\left(x,\left|z_{n}^{1}\right|\right)\right| \\
& -h_{\infty}\left(\left|z_{n}^{1}\right|\right)\left|z_{n}^{1}\right||w| d x \\
& \leq\left[\left(\int_{|x| \leq \rho_{1}}\left|H_{z}\left(x, z_{n}^{1}\right)\right|^{p /(p-1)} d x\right)^{(p-1) / p}\right. \\
& \left.+\left(\int_{|x| \leq \rho_{1}}\left|H_{\infty}^{\prime}\left(z_{n}^{1}\right)\right|^{p /(p-1)} d x\right)^{(p-1) / p}\right] \\
& \cdot\left(\int_{|x| \leq \rho_{1}}|w|^{p} d x\right)^{1 / p}+\varepsilon\left\|z_{n}^{1} w\right\|_{L^{2}\left(\mathbb{R}^{N}, \mathbb{R}^{2}\right)}
\end{aligned}
$$

Hence, we obtain

$$
F^{\prime}\left(z_{n}^{1}\right)=\Phi^{\prime}\left(z_{n}^{1}\right)+\Psi^{\prime}\left(z_{n}^{1}\right)-\Psi_{\infty}^{\prime}\left(z_{n}^{1}\right) \longrightarrow 0 .
$$

In addition, we have

$$
\begin{aligned}
c_{1}+o(1) & =F\left(z_{n}^{1}\right)-\frac{1}{2}\left\langle F^{\prime}\left(z_{n}^{1}\right), z_{n}^{1}\right\rangle \\
& =\int_{\mathbb{R}^{N}} \widetilde{H}_{\infty}\left(z_{n}^{1}\right) d x \geq 0,
\end{aligned}
$$

and $c_{1}=0$ if and only if $z_{n}^{1} \rightarrow 0$ in $E$. In fact, if $c_{1}=0$, then

$$
\int_{\mathbb{R}^{N}} \widetilde{H}_{\infty}\left(z_{n}^{1}\right) d x \rightarrow 0
$$

Hence, using (74) and Hölder inequality $\left(1 / \tau+1 / \tau^{\prime}=1\right)$, we obtain

$$
\begin{aligned}
& \left\|z_{n}^{1}\right\|^{2}=\int_{\mathbb{R}^{N}} h_{\infty}\left(\left|z_{n}^{1}\right|\right) z_{n}^{1}\left(\left(z_{n}^{1}\right)^{+}-\left(z_{n}^{1}\right)^{-}\right) d x+o(1) \\
& \leq \varepsilon\left|z_{n}^{1}\right|_{2}^{2}+C_{\varepsilon}\left(\int_{\mathbb{R}^{N}}\left|z_{n}^{1}\right|^{2}\left|\widetilde{H}_{\infty}\left(z_{n}^{1}\right)\right|^{1 / \tau} d x\right)+o(1) \\
& \leq \varepsilon\left|z_{n}^{1}\right|_{2}^{2} \\
& \quad+C_{\varepsilon}\left(\int_{\mathbb{R}^{N}} \widetilde{H}_{\infty}\left(z_{n}^{1}\right) d x\right)^{1 / \tau}\left(\int_{\mathbb{R}^{N}}\left|z_{n}^{1}\right|^{2 \tau^{\prime}} d x\right)^{1 / \tau^{\prime}} \\
& \quad+o(1) \\
& \leq \varepsilon a_{2}^{2}\left\|z_{n}^{1}\right\|^{2}+a_{2 \tau^{\prime}}^{2} C_{\varepsilon}\left(\int_{\mathbb{R}^{N}} \widetilde{H}_{\infty}\left(z_{n}^{1}\right) d x\right)^{1 / \tau}\left\|z_{n}^{1}\right\|^{2} \\
& \quad+o(1) .
\end{aligned}
$$

This implies that $z_{n}^{1} \rightarrow 0$ in $E$. The inverse is obvious. Now, assume that conclusion (i) is false. Then $c_{1}>0$. For the concentration functions $\left|z_{n}^{1}\right|^{2}$, there are only two cases needed to be considered: vanishing and nonvanishing. If vanishing occurs, by the vanishing lemma and (30)-(31), one has

$$
\begin{array}{r}
\int_{\mathbb{R}^{N}} h_{\infty}\left(\left|z_{n}^{1}\right|\right)\left|z_{n}^{1}\right|^{2} d x=o(1), \\
\int_{\mathbb{R}^{N}} H_{\infty}\left(z_{n}^{1}\right) d x=o(1) .
\end{array}
$$

Hence

$$
\begin{aligned}
c_{1} & =\lim _{n \rightarrow \infty} \int_{\mathbb{R}^{N}} \widetilde{H}_{\infty}\left(z_{n}^{1}\right) d x \\
& =\lim _{n \rightarrow \infty} \int_{\mathbb{R}^{N}} \frac{1}{2} h_{\infty}\left(\left|z_{n}^{1}\right|\right)\left|z_{n}^{1}\right|^{2}-H_{\infty}\left(z_{n}^{1}\right) d x=0 .
\end{aligned}
$$

This is a contradiction. Thus, nonvanishing occurs; that is, there is a sequence $\left\{a_{n}^{1}\right\} \subset \mathbb{R}^{N}$ and constants $r_{1}, \delta_{1}>0$ such that

$$
\left\|z_{n}^{1}\right\|_{L^{2}\left(B\left(a_{n}^{1}, r_{1}\right)\right)} \geq \frac{\delta_{1}}{2}
$$

for large $n$. We may choose $k_{n}^{1} \in \mathbb{Z}^{N}$ and $\bar{z}_{n}^{1}:=k_{n}^{1} * z_{n}^{1}$ such that, passing to a subsequence,

$$
\left\|\bar{z}_{n}^{1}\right\|_{L^{2}\left(B\left(0, r_{1}+(1 / 2) \sqrt{N}\right)\right)} \geq \frac{\delta_{1}}{2}
$$

for all $n$. Note that $F\left(\bar{z}_{n}^{1}\right)=F\left(z_{n}^{1}\right),\left\|\nabla F\left(\bar{z}_{n}^{1}\right)\right\|=\left\|\nabla F\left(z_{n}^{1}\right)\right\|$, and $\left\|\bar{z}_{n}^{1}\right\|=\left\|z_{n}^{1}\right\|$. We know that $\left\{\bar{z}_{n}^{1}\right\}$ is bounded (PS) $)_{c_{1}}$-sequence of 
$F$. Hence, by Lemma 12, passing to a subsequence if necessary, we can assume that $\bar{z}_{n}^{1} \rightarrow \bar{z}_{1} \in \widehat{\mathscr{K}} \backslash\{0\}$,

$$
\begin{aligned}
& F\left(w_{n}^{1}\right) \longrightarrow c-\Phi\left(z_{0}\right)-F\left(\bar{z}_{1}\right), \\
& F^{\prime}\left(w_{n}^{1}\right) \longrightarrow 0
\end{aligned}
$$

as $n \rightarrow \infty$, where $w_{n}^{1}=\bar{z}_{n}^{1}-\bar{z}_{1}$. Note that

$$
\begin{aligned}
c- & \Phi\left(z_{0}\right)-F\left(\bar{z}_{1}\right)+o(1) \\
& =F\left(w_{n}^{1}\right)-\frac{1}{2}\left\langle F^{\prime}\left(w_{n}^{1}\right), w_{n}^{1}\right\rangle=\int_{\mathbb{R}^{N}} \widetilde{H}_{\infty}\left(w_{n}^{1}\right) d x
\end{aligned}
$$

$$
\geq 0 \text {. }
$$

Hence

$$
c-\Phi\left(z_{0}\right)-F\left(\bar{z}_{1}\right) \geq 0 .
$$

Since $\Phi\left(z_{0}\right) \geq 0$ and $F\left(\bar{z}_{1}\right) \geq \widehat{C}$, we obtain that $c \geq \widehat{C}$ and $\widehat{C} \leq F\left(\bar{z}_{1}\right) \leq c-\Phi\left(z_{0}\right)$. There are now two possibilities to consider: $F\left(\bar{z}_{1}\right)<c-\Phi\left(z_{0}\right)$ or $F\left(\bar{z}_{1}\right)=c-\Phi\left(z_{0}\right)$.

Set $c_{1}=c-\Phi\left(z_{0}\right)$. If $c_{2}:=c_{1}-F\left(\bar{z}_{1}\right)=0$, repeat the above arguments but replace $\left\{z_{n}^{1}\right\}$ and $c_{1}$ by $\left\{w_{n}^{1}\right\}$ and $c_{2}$, respectively. We obtain that $c_{2}=0$ if and only if $w_{n}^{1} \rightarrow 0$. Consequently, we obtain that $w_{n}^{1} \rightarrow 0$. Hence the lemma holds with $l=1$, $y_{1}=k_{1} * \bar{z}_{1}$ (where $k_{1} \in \mathbb{Z}^{N}$ is chosen to ensure that $y_{1} \in$ $\widehat{\mathscr{K}} \backslash\{0\})$ and $g_{n}^{1}=-k_{n}^{1}-k_{1}$.

If $c_{2}>0$, then we argue again as above with $\left\{z_{n}^{1}\right\}$ and $c_{1}$ replaced by $\left\{w_{n}\right\}$ and $c_{2}$, respectively, and we obtain $\bar{z}_{2} \in \widehat{\mathscr{K}} \backslash$ $\{0\}$ with $\widehat{C} \leq F\left(\bar{z}_{2}\right) \leq c_{2}$. After at most $[c / \widehat{C}]$ steps, we obtain the desired conclusion.

As a straight consequence of Lemma 28, we have the following.

Lemma 29. $\Phi$ satisfies $(C)_{c}$ condition for all $c<\widehat{C}$.

We are now in a position to complete the proof of Theorem 1 .

Lemma 30. $\Phi$ satisfies $\left(\Phi_{1}\right)$.

Proof. For any $c>0$ and $z \in \Phi_{c}$, using the definition of $\Phi$ and $H(x, z) \geq 0$, one has

$$
0<c \leq \frac{1}{2}\left(\left\|z^{+}\right\|^{2}-\left\|z^{-}\right\|^{2}\right) .
$$

This yields $\left\|z^{-}\right\|<\left\|z^{+}\right\|$, and hence $\|z\|<2\left\|z^{+}\right\|$. $\Phi$ satisfies $\left(\Phi_{1}\right)$.

Proof of Theorem 1. By Lemma 30, $\Phi$ satisfies $\left(\Phi_{1}\right)$. It follows from Lemmas 7, 21, and 22 that $\left(\Phi_{0}\right)$ holds. By Lemma 23, we know that $\left(\Phi_{2}\right)$ holds. Lemma 24 shows that the linking condition of Theorem 6 holds. These, together with Lemma 25, yield $(C)_{c}$-sequence $\left\{z_{n}\right\}$ with $\kappa_{1} \leq c \leq \sup \Phi(Q)<\widehat{C}$ for $\Phi$. By virtue of Lemma 29, we can assume that $z_{n} \rightarrow z$ as $n \rightarrow \infty$. Furthermore, we have $\Phi^{\prime}(z)=0$ and $\Phi(z) \geq \kappa_{1}$. This implies that $\mathscr{K} \backslash\{0\} \neq \emptyset$. The proof is completed.

\section{Summary}

The purpose of this paper is to investigate the existence of nontrivial solutions for a class of Hamiltonian elliptic system in $\mathbb{R}^{N}$ in the case that the nonlinearity may not satisfy the standard Ambrosetti-Rabinowitz condition $\left(H_{0}\right)$. We obtain rather general conditions for the existence of nontrivial solutions, which extend and improve some recent results in the literature. In this paper, since we have no assumption of periodic about nonlinearity, the problem is more difficult and interesting. We will point out that if the following condition is satisfied:

(HP) $H(x, z)$ is 1 -periodic in $x_{i}$ for $i=1, \ldots, N$, then for any $0 \neq a=\left(a_{1}, \ldots, a_{N}\right) \in \mathbb{Z}^{N}$, we have $\Phi(a * z)=$ $\Phi(z),\|\nabla \Phi(a * z)\|=\|\nabla \Phi(z)\|$, and $\|a * z\|=\|z\|$. Hence, combining with Lemmas 7, 21, 22, 23, and 30, similar to the proof of Lemmas 11 and 13, we have the following.

Theorem 31. $\mathscr{K} \backslash\{0\} \neq \emptyset$ and $\inf \{\Phi(z): z \in \mathscr{K} \backslash\{0\}\}>0$ is attained; that is, the problem (HES) has a ground state solution.

\section{Conflicts of Interest}

The authors declare that they have no conflicts of interest regarding the publication of this paper.

\section{Acknowledgments}

This work is supported partially by the National Natural Science Foundation of China (11426037), the Applied Basic Research Programs of Yunnan Province (2012FD060), Outstanding Young Teachers Training Program of Yunnan Province, and Youth Academic and Technical Leaders of Baoshan Municipality.

\section{References}

[1] J.-L. Lions, Optimal Control of Systems Governed by Partial Differential Equations, Springer, Berlin, Germany, 1971.

[2] S. Itô, Diffusion Equations, vol. 114 of Translations of Mathematical Monographs, American Mathematical Society, Providence, RI, USA, 1992.

[3] M. Nagasawa, Schrödinger Equations and Diffusion Theory, vol. 86 of Monographs in Mathematics, Birkhäuser, Basel, Switzerland, 1993.

[4] D. G. De Figueiredo and J. Yang, "Decay, symmetry and existence of solutions of semilinear elliptic systems," Nonlinear Analysis. Theory, Methods \& Applications. An International Multidisciplinary Journal, vol. 33, no. 3, pp. 211-234, 1998.

[5] B. Sirakov, "On the existence of solutions of Hamiltonian elliptic systems in $R^{N}$," Advances in Differential Equations, vol. 5, no. 10-12, pp. 1445-1464, 2000.

[6] F. K. Zhao, L. G. Zhao, and Y. H. Ding, "Multiple solutions for asymptotically linear elliptic systems," Nonlinear Differential Equations and Applications, vol. 15, no. 6, pp. 673-688, 2008.

[7] C. O. Alves, P. C. Carrião, and O. H. Miyagaki, "On the existence of positive solutions of a perturbed Hamiltonian system in $\mathrm{R}^{N}$," Journal of Mathematical Analysis and Applications, vol. 276, no. 2, pp. 673-690, 2002. 
[8] A. I. Ávila and J. Yang, "Multiple solutions of nonlinear elliptic systems," Nonlinear Differential Equations and Applications, vol. 12, no. 4, pp. 459-479, 2005.

[9] A. I. Ávila and J. Yang, "On the existence and shape of least energy solutions for some elliptic systems," Journal of Differential Equations, vol. 191, no. 2, pp. 348-376, 2003.

[10] T. Bartsch and Y. Ding, "Homoclinic solutions of an infinitedimensional Hamiltonian system," Mathematische Zeitschrift, vol. 240, no. 2, pp. 289-310, 2002.

[11] T. Bartsch and D. G. De Figueiredo, "Infinitely many solutions of nonlinear elliptic systems," Nonlinear Differential Equations and Applications, vol. 35, pp. 51-67, 1999.

[12] J. Busca and B. Sirakov, "Symmetry results for semilinear elliptic systems in the whole space," Journal of Differential Equations, vol. 163, no. 1, pp. 41-56, 2000.

[13] A. V. Lair and A. W. Wood, "Existence of entire large positive solutions of semilinear elliptic systems," Journal of Differential Equations, vol. 164, no. 2, pp. 380-394, 2000.

[14] G. Li and J. Yang, "Asymptotically linear elliptic systems," Communications in Partial Differential Equations, vol. 29, no. 56, pp. 925-954, 2004.

[15] A. Pistoia and M. Ramos, "Locating the peaks of the least energy solutions to an elliptic system with Neumann boundary conditions," Journal of Differential Equations, vol. 201, no. 1, pp. 160-176, 2004.

[16] M. Schechter and W. M. Zou, "Homoclinic orbits for Schrödinger systems," Michigan Mathematical Journal, vol. 51, no. 1, pp. 59-71, 2003.

[17] J. Yang, "Nontrivial solutions of semilinear elliptic systems in $R^{N}$," Electronic Journal of Differential Equations, vol. 6, pp. 343$357,2001$.

[18] J. Wang, J. Xu, and F. Zhang, "Existence of solutions for nonperiodic superquadratic Hamiltonian elliptic systems," Nonlinear Analysis. Theory, Methods \& Applications, vol. 72, no. 3-4, pp. 1949-1960, 2010.

[19] F. Zhao and Y. Ding, "On Hamiltonian elliptic systems with periodic or non-periodic potentials," Journal of Differential Equations, vol. 249, no. 12, pp. 2964-2985, 2010.

[20] Y. H. Ding, Variational Methods for Strongly Indefinite Problems, World Scientific Press, Singapore, 2008.

[21] Y. Ding and C. Lee, "Existence and exponential decay of homoclinics in a nonperiodic superquadratic Hamiltonian system," Journal of Differential Equations, vol. 246, no. 7, pp. 2829-2848, 2009.

[22] Y. Ding and J. Wei, "Stationary states of nonlinear Dirac equations with general potentials," Reviews in Mathematical Physics, vol. 20, no. 8, pp. 1007-1032, 2008.

[23] Y. H. Ding and A. Szulkin, "Bound states for semilinear Schrödinger equations with sign-changing potential," Calculus of Variations and Partial Differential Equations, vol. 29, no. 3, pp. 397-419, 2007.

[24] W. Kryszewski and A. Szulkin, "Generalized linking theorem with an application to semilinear Schrödinger equations," Advances in Differential Equations, vol. 3, no. 3, pp. 441-472, 1998.

[25] A. Szulkin and W. Zou, "Homoclinic orbits for asymptotically linear Hamiltonian systems," Journal of Functional Analysis, vol. 187, no. 1, pp. 25-41, 2001.

[26] C. Troestler and M. Willem, "Nontrivial solution of a semilinear Schrödinger equation," Communications in Partial Differential Equations, vol. 21, no. 9-10, pp. 1431-1449, 1996.
[27] M. Willem, Minimax Theorems, vol. 24 of Progress in Nonlinear Differential Equations and their Applications, Birkhäuser, Boston, Mass, USA, 1996.

[28] N. Ackermann, "A superposition principle and multibump solutions of periodic Schrödinger equations," Journal of Functional Analysis, vol. 234, no. 2, pp. 277-320, 2006. 


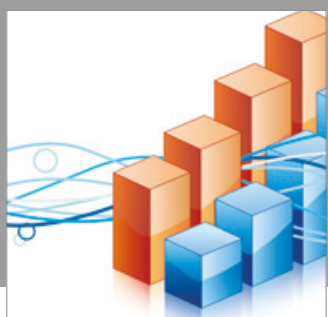

Advances in

Operations Research

vatersals

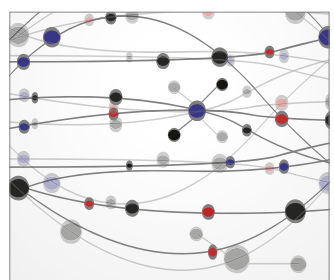

\section{The Scientific} World Journal
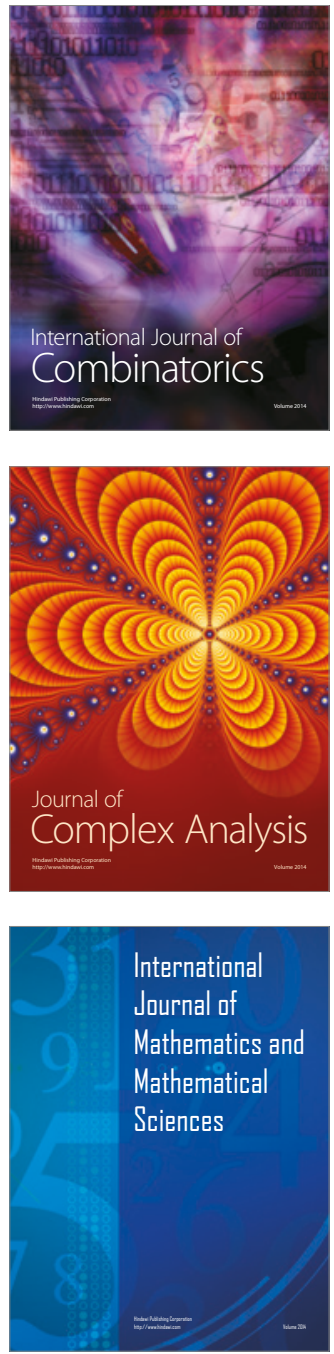
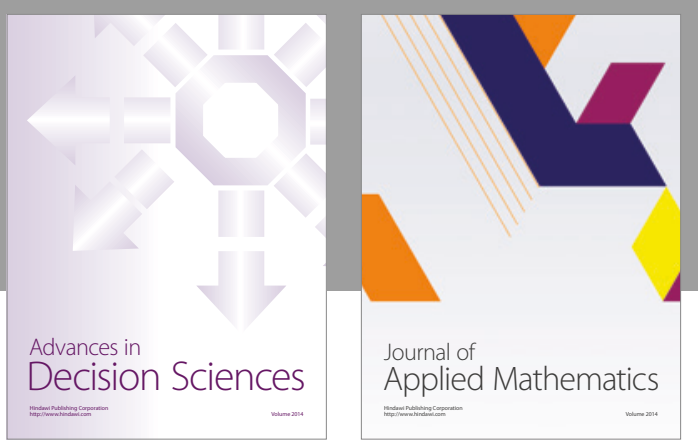

Algebra

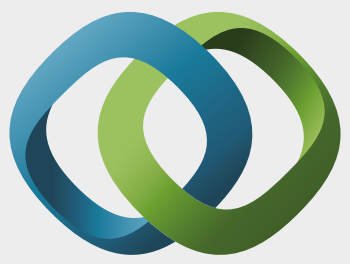

\section{Hindawi}

Submit your manuscripts at

https://www.hindawi.com
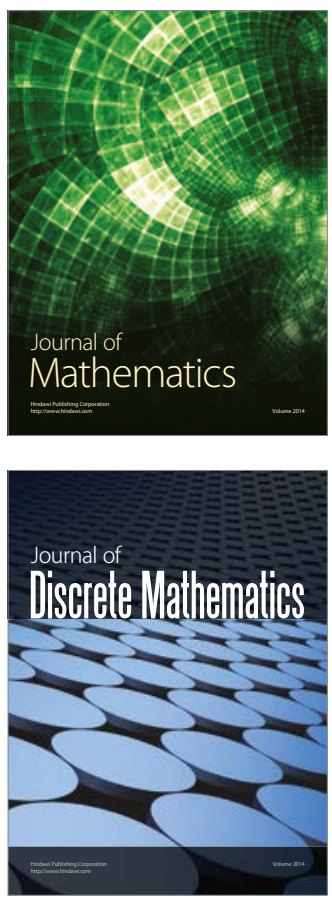

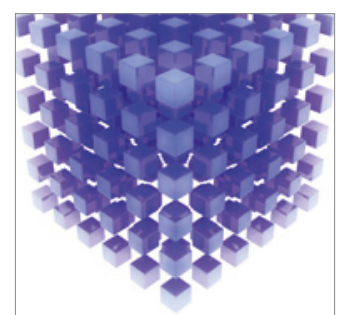

Mathematical Problems in Engineering
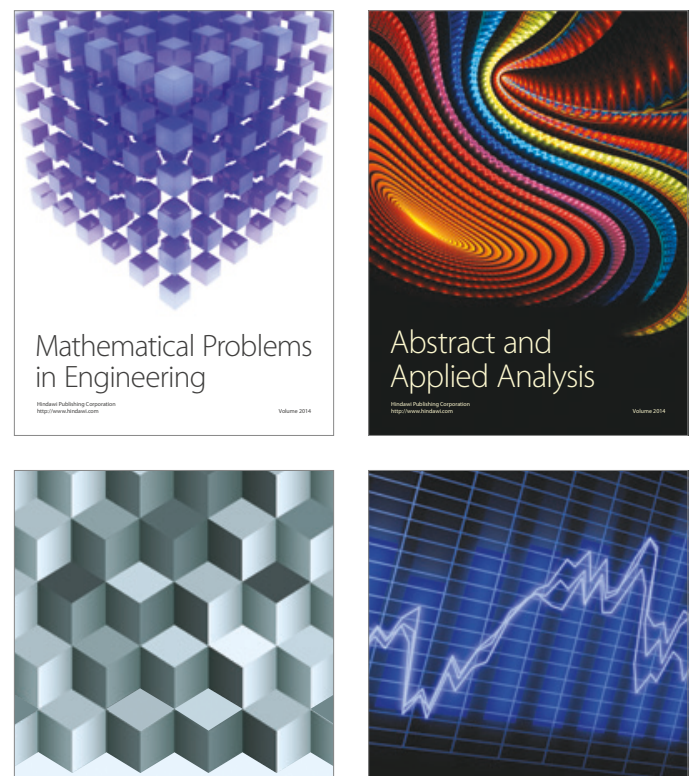

Journal of

Function Spaces

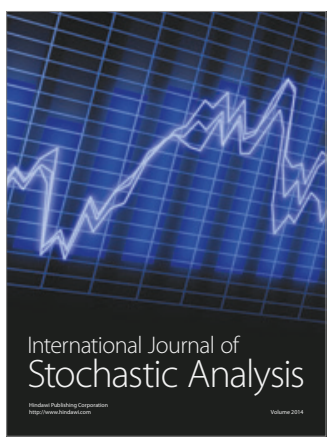

Probability and Statistics
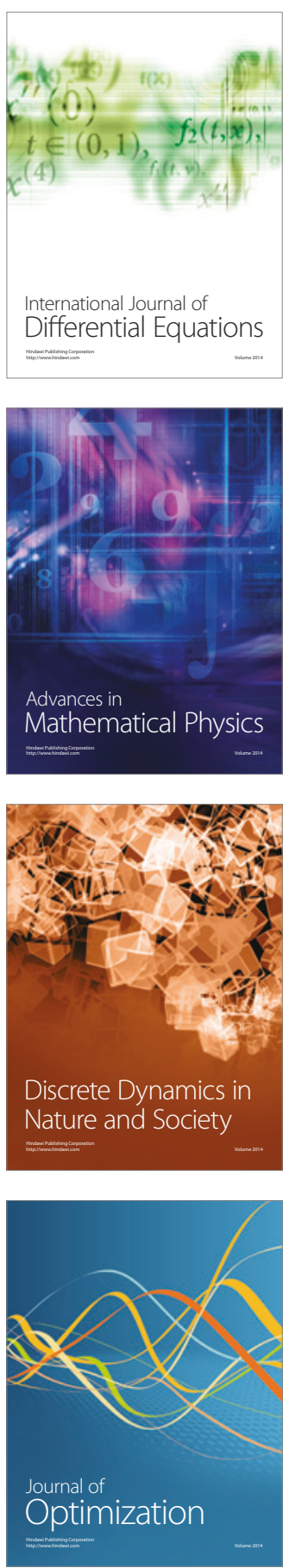